\title{
On Estimation and Prediction for the Inverted Kumaraswamy Distribution Based on General Progressive Censored Samples
}

\author{
Moustafa Mohie El-Din \\ Department of Mathematics, Faculty of Science, Al-Azhar University, Cairo-Egypt. \\ mmmmoheeldin@yahoo.com \\ Mahmoud Abu-Moussa \\ Department of Mathematics, Faculty of Science, Cairo University, Giza-Egypt. \\ mhmoussa@sci.cu.edu.eg
}

\begin{abstract}
In this article, the problem of estimating unknown parameters of the inverted kumaraswamy (IKum) distribution is considered based on general progressive Type-II censored Data. The maximum likelihood (MLE) estimators of the parameters are obtained while the Bayesian estimates are obtained using the squared error loss(SEL) as symmetric loss function. Also we used asymmetric loss functions as the linear-exponential loss (LINEX), generalized entropy (GE) and Al-Bayatti loss function (AL-Bayatti). Lindely's approximation method is used to evaluate the Bayes estimates. We also derived an approximate confidence interval for the parameters of the inverted Kumaraswamy distribution. Two-sample Bayesian prediction intervals are constructed with an illustrative example. Finally, simulation study concerning different sample sizes and different censoring schemes were reported.
\end{abstract}

Keywords: Maximum likelihood and bayesian estimation; General progressive Type-II censored Data; Inverted kumaraswamy distribution; Asymptotic confidence intervals; Two-sample bayesian prediction.

\section{Introduction}

In most of life-testing experiments, the censored samples used when the experimenter wants to terminate the experiment early before all units are failed due to the time limitation and the huge cost of the experiment.Type-I and Type-II are the two basic types of censoring schemes, where in Type-I the experiment is terminated at pre-specified time point and the number of failures is variable, while the experiment under Type-II is terminated after a fixed number of failures. Removing unites at certain time points during the experiment are not allowed in Type-I and Type-II, so Progressive censoring is applicable, such that the experimenter can remove some units at pre-specified time points (Progressive Type-I) or remove units at each failure (Progressive Type-II). For further reading about progressive censoring, see Balakrishnan and Aggarwala (2000) and Balakrishnan (2007) who presented a study on different features of progressive censoring schemes.

Now, suppose that we have $n$ units were placed on a lifetime- experiment, suppose that the first $r$ failures $X_{1: m: n}, X_{2: m: n}, \ldots, X_{r: m: n}$ were not observed and the other $m-r, 0 \leq r<m \leq n$ failures $X_{r+1: m: n}, X_{r+2: m: n}, \ldots, X_{m: m: n}$ are observed. At the $(r+1)$ th failure time $X_{r+1: m: n}, R_{r+1}$ number of surviving units are randomly selected and removed from the test, at the $(r+2)$ th failure time $X_{r+2: m: n}, R_{r+2}$ number of surviving units are also randomly selected and removed, and so on until the $(m)$ th failure $X_{m: m: n}$, the remaining survived unites $R_{m}=n-m-R_{r+1}-R_{r+2}-\ldots-R_{m-1}$ are 
removed from the lifetime-test. The number of removals $R_{i^{\prime}} s, m$ and $r$ are pre-specified integers with $0<R_{i}<n-i$. Then the resulting failures $X_{r+1: m: n}, X_{r+2: m: n}, \ldots, X_{m: m: n}$ are referred to general progressively Type-II censored data. There are some special cases for which the general progressive censoring can reduces to other censoring types, as shown in the following table,

\begin{tabular}{|c|c|c|c|}
\hline $\mathrm{r}$ & $R_{i}$ & $\mathrm{n}, \mathrm{m}$ & Type of censoring \\
\hline $\mathrm{r}>0$ & $R_{i}=0, i=r+1, \ldots, m-1, R_{m}=n-m$ & $m \leq n$ & Type-II doubly censored sample \\
\hline $\mathrm{r}=0$ & $R_{i}>0, i=r+1, \ldots, m-1$ & $m \leq n$ & progressive Type-II right censoring \\
\hline $\mathrm{r}=0$ & $R_{i}=0, i=r+1, \ldots, m-1, R_{m}=n-m$ & $m \leq n$ & conventional Type-II right censoring \\
\hline $\mathrm{r}=0$ & $R_{i}=0, i=r+1, \ldots, m$ & $m=n$ & no censoring (complete sample) \\
\hline
\end{tabular}

Many authors have been studied the general progressive censoring using different lifetime distributions, as, Soliman (2008) make an inference for Pareto model using general progressive censored data. Also Xiuyun and Zaizai (2016) study the Bayesian estimation and prediction for the inverse Weibull distribution under general progressive censoring, while the Characterization for Gompertz distribution based on general progressively type-II right censored order statistics have been studied by Mohie El-Din et. al. (2017).

Bayesian prediction is an important topic in statistical inference where we try to use the previous data to predict the future observations inside the same population with a specified probability. When the unobserved failures belong two the same sample, then the prediction called One-sample Bayesian prediction, while it is called Two-sample Bayesian prediction when we want to predict by a new sample using an old sample. The Bayesian prediction was discussed by many authors based on different distributions with different types of censored samples as Mohie El-Din and Shafay (2013), they study Bayesian prediction intervals based on progressively Type-II censored data. Shafay and Balakrishnan (2012) study the Bayesian prediction intervals based on the Type-I hybrid censored data. Bayesian prediction intervals of generalized order statistics based on multiply Type-II censored data was discussed by Mohie El-Din et al.(2012), they also studied the Bayesian prediction for order statistics from a general class of distributions based on left Type-II censored data, see (2011). Latest Mohie El-Din et al. (2017) study the One-sample Bayesian prediction intervals based on Type-II progressively hybrid censored samples.

In 2017 Abd Al-Fattah et. al. (2017) introduced the inverted Kumaraswamy (IKum) distribution and studied its properties. IKum distribution can be used in long term reliability predictions, producing optimistic predictions of rare events occurring in the right tail of the distribution compared with other distributions.

Let $X$ be a random variable distributed as IKum distribution with shape parameters; $\alpha>0$ and $\beta>0$, denoted by $X \sim \operatorname{IKum}(\alpha, \beta)$. Then the probability density function (pdf), cumulative distribution function (cdf), reliability function (rf)and the hazard rate function (hrf) are given, respectively, as follows

$$
f(x ; \alpha, \beta)=\alpha \beta(1+x)^{-(\alpha+1)}\left(1-(1+x)^{-\alpha}\right)^{\beta-1}, \quad x \geq 0, \alpha, \beta>0,
$$




$$
\begin{aligned}
& F(x ; \alpha, \beta)=\left(1-(1+x)^{-\alpha}\right)^{\beta}=\frac{f(x)}{\alpha \beta}\left((1+x)^{\alpha+1}-(1+x)\right), \\
& R(x)=1-F(x)=1-\left(1-(1+x)^{-\alpha}\right)^{\beta}
\end{aligned}
$$

and

$$
h(x)=\frac{f(x)}{R(x)}=\frac{\alpha \beta(1+x)^{-(\alpha+1)}\left(1-(1+x)^{-\alpha}\right)^{\beta-1}}{1-\left(1-(1+x)^{-\alpha}\right)^{\beta-1}} .
$$

This article is organized as follows. In section 2, the likelihood function and the maximum likelihood estimates of $\alpha$ and $\beta$ are obtained, also the asymptotic confidence intervals are constructed in the same section. In section 3, Bayes estimates for the parameters $\alpha$ and $\beta$ are obtained using four different loss functions (SEL, LINEX, GE and Al-Bayatti). In section 4, the approximation of Bayesian estimates are obtained using Lindley's approximation method. In section 5, a real data example is constructed to compare the proposed methods. In section 6, simulation study is performed to discover the properties of different estimators proposed in this paper. Finally, the paper is concluded in section 7.

\section{Maximum Likelihood Estimation (MLE)}

Suppose that $\mathrm{n}$ randomly selected units have a lifetimes follow $\operatorname{IKum}(\alpha, \beta)$ distribution are put on the lifetime-test at time zero. Based on the general progressively Type-II censoring, then the sample is given by $X_{r+1: m: n}, X_{r+2: m: n}, \ldots, X_{m: m: n}$ with the progressive censoring scheme $R_{r+1}, R_{r+2}, \ldots, R_{m}$. For simple notation, we use $X_{i}$ instead of $X_{i: m: n}$, then $\mathbf{x}=\left(x_{r+1}, x_{r+2}, \ldots, x_{m}\right)$ be the general progressive censored sample. The likelihood function is given by

$$
L(x ; \alpha, \beta)=c\left[F\left(x_{r+1}\right)\right]^{r} \prod_{i=r+1}^{m} f\left(x_{i}\right)\left[1-F\left(x_{i}\right)\right]^{R_{i}} .
$$

Where

$$
\begin{aligned}
& c=\left(\begin{array}{l}
n \\
r
\end{array}\right)(n-r)\left(n-r-R_{r+1}-1\right)\left(n-r-R_{r+1}-R_{r+2}-2\right) \ldots \\
& \times\left(n-r-R_{r+1}-R_{r+2}-\ldots-R_{m-1}-(m-r)+1\right) \\
& =\left(\begin{array}{l}
n \\
r
\end{array}\right)(n-r) \prod_{j=r+2}^{m}\left[n-\sum_{i=r+1}^{j-1} R_{i}-j+1\right] .
\end{aligned}
$$

Using (1) and (2), then the likelihood function becomes

$$
L(x ; \alpha, \beta)=c\left[1-\left(1+x_{r+1}\right)^{-\alpha}\right]^{\beta r} \cdot \prod_{i=r+1}^{m} \alpha \beta\left(1+x_{i}\right)^{-\alpha-1}\left[1-\left(1+x_{i}\right)^{-\alpha}\right]^{\beta-1}\left[1-\left(1-\left(1+x_{i}\right)^{-\alpha}\right)^{\beta}\right]^{R_{i}}
$$

Taking the logarithm for (7), then the log-likelihood function is

$$
\begin{gathered}
l(x ; \alpha, \beta)=\log c+\beta r \log \left[1-\left(1+x_{r+1}\right)^{-\alpha}\right]+\sum_{i=r+1}^{m}\left[\log \alpha+\log \beta-(\alpha+1) \log \left(1+x_{i}\right)\right] \\
+\sum_{i=r+1}^{m}\left[(\beta-1) \log \left[1-\left(1+x_{i}\right)^{-\alpha}\right]+R_{i} \log \left[1-\left(1-\left(1+x_{i}\right)^{-\alpha}\right)^{\beta}\right]\right]
\end{gathered}
$$


By applying the partial derivatives for (8) with respect to $\alpha$ and $\beta$ and putting the derivatives equal to zero, then we get:

$$
\begin{aligned}
& \frac{\partial l}{\partial \alpha}=\frac{\beta r\left(1+x_{r+1}\right)^{-\alpha} \log \left(x_{r+1}+1\right)}{1-\left(1+x_{r+1}\right)^{-\alpha}}-\sum_{i=r+1}^{m} \frac{\beta R_{i}\left(1+x_{i}\right)^{-\alpha} \log \left(x_{i}+1\right)\left(1-\left(1+x_{i}\right)^{-\alpha}\right)^{\beta-1}}{1-\left(1-\left(1+x_{i}\right)^{-\alpha}\right)^{\beta}} \\
& +\sum_{i=r+1}^{m}\left(\frac{1}{\alpha}+\frac{(\beta-1)\left(1+x_{i}\right)^{-\alpha} \log \left(1+x_{i}\right)}{1-\left(1+x_{i}\right)^{-\alpha}}-\log \left(1+x_{i}\right)\right)=0,
\end{aligned}
$$

and

$$
\begin{aligned}
\frac{\partial l}{\partial \beta}= & \log \left(1-\left(x_{r+1}+1\right)^{-\alpha}\right)-\sum_{i=r+1}^{m} \frac{R_{i}\left(1-\left(1+x_{i}\right)^{-\alpha}\right)^{\beta} \log \left(1-\left(x_{i}+1\right)^{-\alpha}\right)}{1-\left(1-\left(x_{i}+1\right)^{-\alpha}\right)^{\beta}} \\
& +\sum_{i=r+1}^{m}\left(\frac{1}{\beta}+\log \left(1-\left(x_{i}+1\right)^{-\alpha}\right)\right)=0,
\end{aligned}
$$

it is obviously that the closed form solution for the parameters $\alpha$ and $\beta$ from the likelihood equations given in (9) and (10) is not possible. However, we can solve these equations by using Newton's iteration method. Let $\hat{\alpha}_{M L}$ and $\hat{\beta}_{M L}$ denote the maximum likelihood estimators of $\alpha$ and $\beta$, respectively. The maximum likelihood estimators for the reliability function $R(x)$ and the hazard function $h(x)$, denoted by $\hat{R}(x)_{M L}$ and $\hat{h}(x)_{M L}$ can be obtained from (3) and (4) by replacing $\alpha$ and $\beta$ by $\hat{\alpha}_{M L}$ and $\hat{\beta}_{M L}$, respectively

\subsection{Observed Fisher Information}

In this subsection, the observed fisher information based on general progressive censoring are observed to construct interval estimates for the parameters of Inverted Kumaraswamy distribution. Using Equations. (9) and (10), we have:

$$
\begin{aligned}
& \frac{\partial^{2} l}{\partial \alpha^{2}}=\sum_{i=r+1}^{m}\left(-\frac{1}{\alpha^{2}}-\frac{(\beta-1)\left(x_{i}+1\right)^{-2 \alpha} \log ^{2}\left(x_{i}+1\right)}{\left(1-\left(x_{i}+1\right)^{-\alpha}\right)^{2}}-\frac{(\beta-1)\left(x_{i}+1\right)^{-\alpha} \log ^{2}\left(x_{i}+1\right)}{1-\left(x_{i}+1\right)^{-\alpha}}\right) \\
& +\sum_{i=r+1}^{m}\left(-\frac{\beta^{2} R_{i}\left(x_{i}+1\right)^{-2 \alpha} \log ^{2}\left(x_{i}+1\right)\left(1-\left(x_{i}+1\right)^{-\alpha}\right)^{2 \beta-2}}{\left(1-\left(1-\left(x_{i}+1\right)^{-\alpha}\right)^{\beta}\right)^{2}}\right) \\
& -\sum_{i=r+1}^{m}\left(\frac{(\beta-1) \beta R_{i}\left(x_{i}+1\right)^{-2 \alpha} \log ^{2}\left(x_{i}+1\right)\left(1-\left(x_{i}+1\right)^{-\alpha}\right)^{\beta-2}}{1-\left(1-\left(x_{i}+1\right)^{-\alpha}\right)^{\beta}}\right)-\frac{\beta r\left(x_{r+1}+1\right)^{\alpha} \log ^{2}\left(x_{r+1}+1\right)}{\left(\left(x_{r+1}+1\right)^{\alpha}-1\right)^{2}} \\
& +\sum_{i=r+1}^{m}\left(\frac{\beta R_{i}\left(x_{i}+1\right)^{-\alpha} \log ^{2}\left(x_{i}+1\right)\left(1-\left(x_{i}+1\right)^{-\alpha}\right)^{\beta-1}}{1-\left(1-\left(x_{i}+1\right)^{-\alpha}\right)^{\beta}}\right) \\
& \frac{\partial^{2} l}{\partial \beta^{2}}=\sum_{i=r+1}^{m}\left(-\frac{R_{i}\left(1-\left(x_{i}+1\right)^{-\alpha}\right)^{\beta} \log ^{2}\left(1-\left(x_{i}+1\right)^{-\alpha}\right)}{1-\left(1-\left(x_{i}+1\right)^{-\alpha}\right)^{\beta}}\right)
\end{aligned}
$$




$$
-\sum_{i=r+1}^{m}\left(\frac{R_{i}\left(1-\left(x_{i}+1\right)^{-\alpha}\right)^{2 \beta} \log ^{2}\left(1-\left(x_{i}+1\right)^{-\alpha}\right)}{\left(1-\left(1-\left(x_{i}+1\right)^{-\alpha}\right)^{\beta}\right)^{2}}\right)+\frac{r-m}{\beta^{2}}
$$

and

$$
\begin{aligned}
& \frac{\partial^{2} l}{\partial \alpha \partial \beta}=\frac{r \log \left(x_{r+1}+1\right)}{\left(x_{r+1}+1\right)^{\alpha}-1}+\sum_{i=r+1}^{m}\left(-\frac{R_{i}\left(x_{i}+1\right)^{-\alpha} \log \left(x_{i}+1\right)\left(1-\left(x_{i}+1\right)^{-\alpha}\right)^{\beta-1}}{1-\left(1-\left(x_{i}+1\right)^{-\alpha}\right)^{\beta}}\right) \\
& -\sum_{i=r+1}^{m}\left(\frac{\beta R_{i}\left(x_{i}+1\right)^{-\alpha} \log \left(x_{i}+1\right)\left(1-\left(x_{i}+1\right)^{-\alpha}\right)^{\beta-1} \log \left(1-\left(x_{i}+1\right)^{-\alpha}\right)}{1-\left(1-\left(x_{i}+1\right)^{-\alpha}\right)^{\beta}}\right) \\
& -\sum_{i=r+1}^{m}\left(\frac{\beta R_{i}\left(x_{i}+1\right)^{-\alpha} \log \left(x_{i}+1\right)\left(1-\left(x_{i}+1\right)^{-\alpha}\right)^{2 \beta-1} \log \left(1-\left(x_{i}+1\right)^{-\alpha}\right)}{\left(1-\left(1-\left(x_{i}+1\right)^{-\alpha}\right)^{\beta}\right)^{2}}\right)+\sum_{i=r+1}^{m} \frac{\left(x_{i}+1\right)^{-\alpha} \log \left(x_{i}+1\right)}{1-\left(x_{i}+1\right)^{-\alpha}} .
\end{aligned}
$$

Then the asymptotic Variance-Covariance matrix is the inverse of the Fisher information matrix, which is given by,

$$
\hat{V}=\left\{\left[\begin{array}{cc}
\frac{-\partial^{2} l}{\partial \alpha^{2}} & \frac{-\partial^{2} l}{\partial \alpha \partial \beta} \\
\frac{-\partial^{2} l}{\partial \beta \partial \alpha} & \frac{-\partial^{2} l}{\partial \beta^{2}}
\end{array}\right]^{-1}\right\}_{\hat{\alpha}_{M L}, \hat{\beta}_{M L}}=\left[\begin{array}{cc}
\hat{\operatorname{Var}}(\hat{\alpha}) & \hat{\operatorname{Cov}}(\hat{\alpha}, \hat{\beta}) \\
\hat{\operatorname{Cov}}(\hat{\alpha}, \hat{\beta}) & \hat{\operatorname{Var}}(\hat{\beta})
\end{array}\right] .
$$

Then the asymptotic confidence intervals for the parameters $\alpha$ and $\beta$ is given by,

$$
\hat{\alpha} \pm Z_{\gamma / 2} \sqrt{\hat{\operatorname{Var}}(\hat{\alpha})} \quad \text { and } \hat{\beta} \pm Z_{\gamma / 2} \sqrt{\hat{\operatorname{Var}}(\hat{\beta})} \text {. }
$$

with $100(1-\gamma) \%$ confidence degree; where $Z_{\gamma / 2}$ is obtained from the table of the standard normal distribution.

\section{Bayesian Estimation}

In this section, we derive the Bayesian estimates for the parameters $\alpha$ and $\beta$ of the Inverted Kumaraswamy distribution $\operatorname{IKum}(\alpha, \beta)$ based on general progressive Type-II censoring using four different loss functions. The squared error loss function (SEL) which is defined as symmetric loss function, given by:

$$
L_{1}(\theta, \hat{\theta})=(\hat{\theta}-\theta)^{2},
$$

where $\hat{\theta}$ is an estimate of $\theta$. The Bayes estimate for $\theta$ under the loss function $L_{1}$ is the posterior mean $\hat{\theta}_{B S}=E[\theta \mid \underline{x}]$, the second loss function is the linear exponential (LINEX) loss function, it is an asymmetric loss function and defined as

$$
L_{2}(\theta, \hat{\theta})=e^{h(\hat{\theta}-\theta)}-h(\hat{\theta}-\theta)-1, \quad h \neq 0,
$$

The Bayes estimate for any parameter $\theta$ under the loss function $L_{2}$ is given as,

$$
\hat{\theta}_{B L}=-\frac{1}{h} \log \left[E\left(e^{-h \theta} \mid \underline{x}\right)\right],
$$

the third asymmetric loss function is the generalized entropy function which is given by, 


$$
L_{3}(\theta, \hat{\theta})=\left(\frac{\hat{\theta}}{\theta}\right)^{q}-q \log \left(\frac{\hat{\theta}}{\theta}\right)-1, q \neq 0,
$$

and the Bayes estimate under $L_{3}$ is given by,

$$
\hat{\theta}_{B E}=\left(E\left[\theta^{-q} \mid \underline{x}\right]\right)^{-1 / q},
$$

Finally, we have Al-Bayatti loss function $L_{4}$ which introduced by Al-Bayatti (2002), and given by,

$$
L_{4}(\theta, \hat{\theta})=\theta^{c}(\hat{\theta}-\theta)^{2},
$$

While the Bayes estimate under $L_{4}$ is given by,

$$
\hat{\theta}_{B B}=\frac{\int_{0}^{\infty} \theta^{c+1} \pi(\theta \mid \underline{x}) d \theta}{\int_{0}^{\infty} \theta^{c} \pi(\theta \mid \underline{x}) d \theta}=\frac{E\left(\theta^{c+1} \mid \underline{x}\right)}{E\left(\theta^{c} \mid \underline{x}\right)} .
$$

Assume that the parameters $\alpha$ and $\beta$ are independent variables having Weibull prior distributions $\pi_{1}(\alpha)$ and $\pi_{2}(\beta)$ respectively.

$$
\pi_{1}(\alpha) \propto \alpha^{a_{1}-1} e^{-b_{1} \alpha^{a_{1}}} \quad \text { and } \quad \pi_{2}(\beta) \propto \beta^{a_{2}-1} e^{-b_{2} \beta^{a_{2}}},
$$

where $a_{1}, b_{1}, a_{2}, b_{2}>0$ are the hyper parameters of the priors. Using (7) and (16), then the posterior distribution of $\alpha$ and $\beta$ is obtained as

$$
\begin{aligned}
& \pi^{*}(\alpha, \beta \mid \underline{X})=\frac{1}{k} L(\alpha, \beta) \cdot \pi_{1}(\alpha) \pi_{2}(\beta) \\
& =\frac{1}{k}\left[1-\left(1+x_{r+1}\right)^{-\alpha}\right]^{\beta r} \alpha^{m-r+a_{1}-1} \beta^{m-r+a_{2}-1} e^{-\left(b_{1} \alpha^{a_{1}}+b_{2} \beta^{a_{2}}\right)} \\
& \times \prod_{i=r+1}^{m}\left(1+x_{i}\right)^{-\alpha-1}\left[1-\left(1+x_{i}\right)^{-\alpha}\right]^{\beta-1}\left[1-\left(1-\left(1+x_{i}\right)^{-\alpha}\right)^{\beta}\right]^{R_{i}},
\end{aligned}
$$

where

$$
\begin{aligned}
& k=\int_{0}^{\infty} \int_{0}^{\infty} L(\alpha, \beta) . \pi_{1}(\alpha) \pi_{2}(\beta) d \alpha d \beta \\
& =\int_{0}^{\infty} \int_{0}^{\infty}\left[1-\left(1+x_{r+1}\right)^{-\alpha}\right]^{\beta r} \alpha^{m-r+a_{1}-1} \beta^{m-r+a_{2}-1} e^{-\left(b_{1} \alpha^{a_{1}}+b_{2} \beta^{a_{2}}\right)} \\
& \times \prod_{i=r+1}^{m}\left(1+x_{i}\right)^{-\alpha-1}\left[1-\left(1+x_{i}\right)^{-\alpha}\right]^{\beta-1}\left[1-\left(1-\left(1+x_{i}\right)^{-\alpha}\right)^{\beta}\right]^{R_{i}} d \alpha d \beta .
\end{aligned}
$$

The Bayes estimates for the parameters $\alpha$ and $\beta$ under the squared error loss function $L_{1}$ are given by,

$$
\begin{aligned}
& \hat{\alpha}_{B S}=E[\alpha \mid \underline{x}]=\frac{1}{k} \int_{0}^{\infty} \int_{0}^{\infty}\left[1-\left(1+x_{r+1}\right)^{-\alpha}\right]^{\beta r} \alpha^{m-r+a_{1}} \beta^{m-r+a_{2}-1} e^{-\left(b_{1} \alpha^{a_{1}}+b_{2} \beta^{a_{2}}\right)} \\
& \times \prod_{i=r+1}^{m}\left(1+x_{i}\right)^{-\alpha-1}\left[1-\left(1+x_{i}\right)^{-\alpha}\right]^{\beta-1}\left[1-\left(1-\left(1+x_{i}\right)^{-\alpha}\right)^{\beta}\right]^{R_{i}} d \alpha d \beta . \\
& \hat{\beta}_{B S}=E[\beta \mid \underline{x}]=\frac{1}{k} \int_{0}^{\infty} \int_{0}^{\infty}\left[1-\left(1+x_{r+1}\right)^{-\alpha}\right]^{\beta r} \alpha^{m-r+a_{1}-1} \beta^{m-r+a_{2}} e^{-\left(b_{1} \alpha^{a_{1}}+b_{2} \beta^{a_{2}}\right)}
\end{aligned}
$$




$$
\times \prod_{i=r+1}^{m}\left(1+x_{i}\right)^{-\alpha-1}\left[1-\left(1+x_{i}\right)^{-\alpha}\right]^{\beta-1}\left[1-\left(1-\left(1+x_{i}\right)^{-\alpha}\right)^{\beta}\right]^{R_{i}} d \alpha d \beta
$$

For the LINEX loss function $L_{2}$, the Bayes estimates for $\alpha$ and $\beta$ are given by,

$$
\hat{\alpha}_{B L}=-\frac{1}{h} \log \left(E\left[e^{-h \alpha} \mid \underline{x}\right]\right), h \neq 0
$$

where

$$
\begin{aligned}
& E\left[e^{-h \alpha} \mid \underline{x}\right]=\frac{1}{k} \int_{0}^{\infty} \int_{0}^{\infty}\left[1-\left(1+x_{r+1}\right)^{-\alpha}\right]^{\beta r} \alpha^{m-r+a_{1}-1} \beta^{m-r+a_{2}-1} e^{-\left(h+b_{1} \alpha^{a_{1}-1}\right) \alpha-b_{2} \beta^{a_{2}}} \\
& \times \prod_{i=r+1}^{m}\left(1+x_{i}\right)^{-\alpha-1}\left[1-\left(1+x_{i}\right)^{-\alpha}\right]^{\beta-1}\left[1-\left(1-\left(1+x_{i}\right)^{-\alpha}\right)^{\beta}\right]^{R_{i}} d \alpha d \beta \\
& \hat{\beta}_{B L}=-\frac{1}{h} \log \left(E\left[e^{-h \beta} \mid \underline{x}\right]\right), h \neq 0
\end{aligned}
$$

where

$$
\begin{aligned}
& E\left[e^{-h \beta} \mid \underline{x}\right]=\frac{1}{k} \int_{0}^{\infty} \int_{0}^{\infty}\left[1-\left(1+x_{r+1}\right)^{-\alpha}\right]^{\beta r} \alpha^{m-r+a_{1}-1} \beta^{m-r+a_{2}-1} e^{-b_{1} \alpha^{a_{1}}-\left(h+b_{2} \beta^{a_{2}-1}\right) \beta} \\
& \times \prod_{i=r+1}^{m}\left(1+x_{i}\right)^{-\alpha-1}\left[1-\left(1+x_{i}\right)^{-\alpha}\right]^{\beta-1}\left[1-\left(1-\left(1+x_{i}\right)^{-\alpha}\right)^{\beta}\right]^{R_{i}} d \alpha d \beta .
\end{aligned}
$$

The Bayes estimates for $\alpha$ and $\beta$ depending on the generalized entropy loss function $L_{3}$ is given by,

where

$$
\hat{\alpha}_{B E}=E\left(\alpha^{-q} \mid \underline{x}\right)^{-1 / q}
$$

$$
\begin{aligned}
& E\left[\alpha^{-q} \mid \underline{x}\right]=\frac{1}{k} \int_{0}^{\infty} \int_{0}^{\infty}\left[1-\left(1+x_{r+1}\right)^{-\alpha}\right]^{\beta r} \alpha^{m-r+a_{1}-q-1} \beta^{m-r+a_{2}-1} e^{-\left(b_{1} \alpha^{a_{1}}+b_{2} \beta^{a_{2}}\right)} \\
& \times \prod_{i=r+1}^{m}\left(1+x_{i}\right)^{-\alpha-1}\left[1-\left(1+x_{i}\right)^{-\alpha}\right]^{\beta-1}\left[1-\left(1-\left(1+x_{i}\right)^{-\alpha}\right)^{\beta}\right]^{R_{i}} d \alpha d \beta \\
& \hat{\beta}_{B E}=E\left(\beta^{-q} \mid \underline{x}\right)^{-1 / q}
\end{aligned}
$$

where

$$
\begin{aligned}
& E\left(\beta^{-q} \mid \underline{x}\right)=\frac{1}{k} \int_{0}^{\infty} \int_{0}^{\infty}\left[1-\left(1+x_{r+1}\right)^{-\alpha}\right]^{\beta r} \alpha^{m-r+a_{1}-1} \beta^{m-r+a_{2}-q-1} e^{-\left(b_{1} \alpha^{a_{1}}+b_{2} \beta^{a_{2}}\right)} \\
& \times \prod_{i=r+1}^{m}\left(1+x_{i}\right)^{-\alpha-1}\left[1-\left(1+x_{i}\right)^{-\alpha}\right]^{\beta-1}\left[1-\left(1-\left(1+x_{i}\right)^{-\alpha}\right)^{\beta}\right]^{R_{i}} d \alpha d \beta .
\end{aligned}
$$

Finally, the Bayes estimates for $\alpha$ and $\beta$ depending on Al-Bayatti loss function $L_{4}$ is given by,

$$
\hat{\alpha}_{B B}=\frac{E\left(\alpha^{c+1} \mid \underline{x}\right)}{E\left(\alpha^{c} \mid \underline{x}\right)},
$$

where

$$
E\left(\alpha^{A} \mid \underline{x}\right)=\int_{0}^{\infty} \int_{0}^{\infty}\left[1-\left(1+x_{r+1}\right)^{-\alpha}\right]^{\beta r} \alpha^{m-r+a_{1}-1+A} \beta^{m-r+a_{2}-1} e^{-\left(b_{1} \alpha^{a_{1}}+b_{2} \beta^{a} a^{2}\right.}
$$




$$
\times \prod_{i=r+1}^{m}\left(1+x_{i}\right)^{-\alpha-1}\left[1-\left(1+x_{i}\right)^{-\alpha}\right]^{\beta-1}\left[1-\left(1-\left(1+x_{i}\right)^{-\alpha}\right)^{\beta}\right]^{R_{i}} d \alpha d \beta .
$$

where $A=c, c+1$ and $\hat{\beta}_{B B}$ is given by,

$$
\hat{\beta}_{B B}=\frac{E\left(\beta^{c+1} \mid \underline{x}\right)}{E\left(\beta^{c} \mid \underline{x}\right)},
$$

Unfortunately, all estimates have the form of ratio of two integrals, and the closed forms for these integrals are not obtained. Therefore, the approximated values for these estimates are computed using the Lindley approximation method.

\section{Lindley approximation method}

Lindley (1980)was discussed an approximate Bayesian method. His method used to obtain an approximate for a ratio of two integrals. Suppose $u(\alpha, \beta)$ is a function of $\alpha$ and $\beta, l(\alpha, \beta)$ is the logarithm of the likelihood function mentioned in (8) and $\rho(\alpha, \beta)=\log \pi(\alpha, \beta)=\log \left(\pi_{1}(\alpha) \pi_{2}(\beta)\right)$, then the Lindley method defined as,

$$
\begin{aligned}
& E(u(\alpha, \beta) \mid \underline{x})=\frac{\int_{0}^{\infty} \int_{0}^{\infty} u(\alpha, \beta) e^{l(\alpha, \beta l \underline{x})+\rho(\alpha, \beta)} d \alpha d \beta}{\int_{0}^{\infty} \int_{0}^{\infty} e^{l(\alpha, \beta l \underline{x})+\rho(\alpha, \beta)} d \alpha d \beta} \\
& \cong u(\hat{\alpha}, \hat{\beta})+0.5 \sum\left(\hat{u}_{i j}+2 \hat{u}_{i} \hat{\rho}_{j}\right) \hat{\sigma}_{i j}+0.5 \sum \hat{l}_{i j k} \hat{u}_{i} \hat{\sigma}_{i j} \hat{\sigma}_{k i} \\
& =u(\hat{\alpha}, \hat{\beta})+0.5\left[\left(\hat{u}_{\alpha \alpha}+2 \hat{u}_{\alpha} \hat{\rho}_{\alpha}\right) \hat{\sigma}_{\alpha \alpha}+\left(\hat{u}_{\beta \alpha}+2 \hat{u}_{\beta} \hat{\rho}_{\alpha}\right) \hat{\sigma}_{\beta \alpha}\right. \\
& \left.+\left(\hat{u}_{\alpha \beta}+2 \hat{u}_{\alpha} \hat{\rho}_{\beta}\right) \hat{\sigma}_{\alpha \beta}+\left(\hat{u}_{\beta \beta}+2 \hat{u}_{\beta} \hat{\rho}_{\beta}\right) \hat{\sigma}_{\beta \beta}\right] \\
& +0.5\left[\hat{u}_{\alpha} \hat{\sigma}_{\alpha \alpha}\left(\hat{l}_{\alpha \alpha \alpha} \hat{\sigma}_{\alpha \alpha}+\hat{l}_{\alpha \alpha \beta} \hat{\sigma}_{\beta \alpha}+\hat{l}_{\alpha \beta \alpha} \hat{\sigma}_{\alpha \beta}\right)+\hat{u}_{\alpha} \hat{l}_{\alpha \beta \beta} \hat{\sigma}_{\alpha \beta}^{2}\right. \\
& \left.+\hat{u}_{\beta} \hat{\sigma}_{\alpha \beta}\left(\hat{l}_{\beta \beta \alpha} \hat{\sigma}_{\beta \beta}+\hat{l}_{\beta \alpha \beta} \hat{\sigma}_{\beta \beta}+\hat{l}_{\beta \alpha \alpha} \hat{\sigma}_{\beta \alpha}\right)+\hat{u}_{\beta} \hat{l}_{\beta \beta \beta} \hat{\sigma}_{\beta \beta}^{2}\right]
\end{aligned}
$$

where $\hat{\alpha}$ and $\hat{\beta}$ are the MLE estimators of $\alpha$ and $\beta$, respectively. Also, $u_{i j}$ is the second derivative of the function $u$ with respect to $i$ and $j$, i.e. $\hat{u}_{\beta \beta}$ is MLE of the second derivative of $u(\alpha, \beta)$ with respect to $\beta$. while the other terms are obtained as follows,

$$
\begin{aligned}
& \hat{l}_{\alpha \alpha \alpha}=\left.\frac{\partial^{3} l}{\partial \alpha^{3}}\right|_{\alpha=\hat{\alpha}, \beta=\hat{\beta}}=\frac{\beta r\left(x_{r+1}+1\right)^{\alpha}\left(\left(x_{r+1}+1\right)^{\alpha}+1\right) \log ^{3}\left(x_{r+1}+1\right)}{\left(\left(x_{r+1}+1\right)^{\alpha}-1\right)^{3}} \\
& +\sum_{i=r+1}^{m}\left(\frac{2}{\alpha^{3}}+\frac{2(\beta-1)\left(x_{i}+1\right)^{-3 \alpha} \log ^{3}\left(x_{i}+1\right)}{\left(1-\left(x_{i}+1\right)^{-\alpha}\right)^{3}}+\frac{3(\beta-1)\left(x_{i}+1\right)^{-2 \alpha} \log ^{3}\left(x_{i}+1\right)}{\left(1-\left(x_{i}+1\right)^{-\alpha}\right)^{2}}\right. \\
& \left.+\frac{(\beta-1)\left(x_{i}+1\right)^{-\alpha} \log ^{3}\left(x_{i}+1\right)}{1-\left(x_{i}+1\right)^{-\alpha}}\right)+\sum_{i=r+1}^{m}\left(-\frac{2 \beta^{3} R_{i}\left(x_{i}+1\right)^{-3 \alpha} \log ^{3}\left(x_{i}+1\right)\left(1-\left(x_{i}+1\right)^{-\alpha}\right)^{3 \beta-3}}{\left(1-\left(1-\left(x_{i}+1\right)^{-\alpha}\right)^{\beta}\right)^{3}}\right. \\
& -\frac{(\beta-1) \beta^{2} R_{i}\left(x_{i}+1\right)^{-3 \alpha} \log ^{3}\left(x_{i}+1\right)\left(1-\left(x_{i}+1\right)^{-\alpha}\right)^{2 \beta-3}}{\left(1-\left(1-\left(x_{i}+1\right)^{-\alpha}\right)^{\beta}\right)^{2}}
\end{aligned}
$$




$$
\begin{aligned}
& -\frac{\beta^{2}(2 \beta-2) R_{i}\left(x_{i}+1\right)^{-3 \alpha} \log ^{3}\left(x_{i}+1\right)\left(1-\left(x_{i}+1\right)^{-\alpha}\right)^{2 \beta-3}}{\left(1-\left(1-\left(x_{i}+1\right)^{-\alpha}\right)^{\beta}\right)^{2}} \\
& +\frac{3 \beta^{2} R_{i}\left(x_{i}+1\right)^{-2 \alpha} \log ^{3}\left(x_{i}+1\right)\left(1-\left(x_{i}+1\right)^{-\alpha}\right)^{2 \beta-2}}{\left(1-\left(1-\left(x_{i}+1\right)^{-\alpha}\right)^{\beta}\right)^{2}} \\
& -\frac{(\beta-2)(\beta-1) \beta R_{i}\left(x_{i}+1\right)^{-3 \alpha} \log ^{3}\left(x_{i}+1\right)\left(1-\left(x_{i}+1\right)^{-\alpha}\right)^{\beta-3}}{1-\left(1-\left(x_{i}+1\right)^{-\alpha}\right)^{\beta}} \\
& +\frac{3(\beta-1) \beta R_{i}\left(x_{i}+1\right)^{-2 \alpha} \log ^{3}\left(x_{i}+1\right)\left(1-\left(x_{i}+1\right)^{-\alpha}\right)^{\beta-2}}{1-\left(1-\left(x_{i}+1\right)^{-\alpha}\right)^{\beta}} \\
& \left.-\frac{\beta R_{i}\left(x_{i}+1\right)^{-\alpha} \log ^{3}\left(x_{i}+1\right)\left(1-\left(x_{i}+1\right)^{-\alpha}\right)^{\beta-1}}{1-\left(1-\left(x_{i}+1\right)^{-\alpha}\right)^{\beta}}\right) \text {, } \\
& \hat{l}_{\beta \beta \beta}=\left.\frac{\partial^{3} l}{\partial \beta^{3}}\right|_{\alpha=\hat{\alpha}, \beta=\hat{\beta}}=\frac{2(m-r)}{\beta^{3}}+\sum_{i=r+1}^{m}\left(-\frac{2 R_{i}\left(1-\left(x_{i}+1\right)^{-\alpha}\right)^{3 \beta} \log ^{3}\left(1-\left(x_{i}+1\right)^{-\alpha}\right)}{\left(1-\left(1-\left(x_{i}+1\right)^{-\alpha}\right)^{\beta}\right)^{3}}\right. \\
& \left.-\frac{3 R_{i}\left(1-\left(x_{i}+1\right)^{-\alpha}\right)^{2 \beta} \log ^{3}\left(1-\left(x_{i}+1\right)^{-\alpha}\right)}{\left(1-\left(1-\left(x_{i}+1\right)^{-\alpha}\right)^{\beta}\right)^{2}}-\frac{R_{i}\left(1-\left(x_{i}+1\right)^{-\alpha}\right)^{\beta} \log ^{3}\left(1-\left(x_{i}+1\right)^{-\alpha}\right)}{1-\left(1-\left(x_{i}+1\right)^{-\alpha}\right)^{\beta}}\right) \text {, }
\end{aligned}
$$

Also,

$$
\begin{aligned}
& \hat{l}_{\alpha \beta \alpha}=\hat{l}_{\beta \alpha \alpha}=\left.\frac{\partial^{3} l}{\partial \beta \alpha^{2}}\right|_{\alpha=\hat{\alpha}, \beta=\hat{\beta}}=-\frac{r\left(x_{r+1}+1\right)^{\alpha} \log ^{2}\left(x_{r+1}+1\right)}{\left(\left(x_{r+1}+1\right)^{\alpha}-1\right)^{2}} \\
& +\sum_{i=r+1}^{m}\left(-\frac{\left(x_{i}+1\right)^{-2 \alpha} \log ^{2}\left(x_{i}+1\right)}{\left(1-\left(x_{i}+1\right)^{-\alpha}\right)^{2}}-\frac{\left(x_{i}+1\right)^{-\alpha} \log ^{2}\left(x_{i}+1\right)}{1-\left(x_{i}+1\right)^{-\alpha}}\right) \\
& +\sum_{i=r+1}^{m}\left(-\frac{2 \beta^{2} R_{i}\left(x_{i}+1\right)^{-2 \alpha} \log ^{2}\left(x_{i}+1\right)\left(1-\left(x_{i}+1\right)^{-\alpha}\right)^{\beta-2} \log \left(1-\left(x_{i}+1\right)^{-\alpha}\right)}{\left(1-\left(1-\left(x_{i}+1\right)^{-\alpha}\right)^{\beta}\right)^{3}}\right. \\
& -\frac{2 \beta R_{i}\left(x_{i}+1\right)^{-2 \alpha} \log ^{2}\left(x_{i}+1\right)\left(1-\left(x_{i}+1\right)^{-\alpha}\right)^{2 \beta-2}}{\left(1-\left(1-\left(x_{i}+1\right)^{-\alpha}\right)^{\beta}\right)^{2}} \\
& -\frac{\beta^{2} R_{i}\left(x_{i}+1\right)^{-2 \alpha} \log ^{2}\left(x_{i}+1\right)\left(1-\left(x_{i}+1\right)^{-\alpha}\right)^{2 \beta-2} \log \left(1-\left(x_{i}+1\right)^{-\alpha}\right)}{\left(1-\left(1-\left(x_{i}+1\right)^{-\alpha}\right)^{\beta}\right)^{2}} \\
& -\frac{\beta(2 \beta-1) R_{i}\left(x_{i}+1\right)^{-2 \alpha} \log ^{2}\left(x_{i}+1\right)\left(1-\left(x_{i}+1\right)^{-\alpha}\right)^{2 \beta-2} \log \left(1-\left(x_{i}+1\right)^{-\alpha}\right)}{\left(1-\left(1-\left(x_{i}+1\right)^{-\alpha}\right)^{\beta}\right)^{2}} \\
& +\frac{\beta R_{i}\left(x_{i}+1\right)^{-\alpha} \log ^{2}\left(x_{i}+1\right)\left(1-\left(x_{i}+1\right)^{-\alpha}\right)^{2 \beta-1} \log \left(1-\left(x_{i}+1\right)^{-\alpha}\right)}{\left.\left(1-\left(x_{i}+1\right)^{-\alpha}\right)^{\beta}\right)^{2}}
\end{aligned}
$$




$$
\begin{aligned}
& -\frac{(\beta-1) R_{i}\left(x_{i}+1\right)^{-2 \alpha} \log ^{2}\left(x_{i}+1\right)\left(1-\left(x_{i}+1\right)^{-\alpha}\right)^{\beta-2}}{1-\left(1-\left(x_{i}+1\right)^{-\alpha}\right)^{\beta}} \\
& -\frac{\beta R_{i}\left(x_{i}+1\right)^{-2 \alpha} \log ^{2}\left(x_{i}+1\right)\left(1-\left(x_{i}+1\right)^{-\alpha}\right)^{\beta-2}}{1-\left(1-\left(x_{i}+1\right)^{-\alpha}\right)^{\beta}} \\
& -\frac{(\beta-1) \beta R_{i}\left(x_{i}+1\right)^{-2 \alpha} \log ^{2}\left(x_{i}+1\right)\left(1-\left(x_{i}+1\right)^{-\alpha}\right)^{\beta-2} \log \left(1-\left(x_{i}+1\right)^{-\alpha}\right)}{1-\left(1-\left(x_{i}+1\right)^{-\alpha}\right)^{\beta}} \\
& +\frac{R_{i}\left(x_{i}+1\right)^{-\alpha} \log ^{2}\left(x_{i}+1\right)\left(1-\left(x_{i}+1\right)^{-\alpha}\right)^{\beta-1}}{1-\left(1-\left(x_{i}+1\right)^{-\alpha}\right)^{\beta}} \\
& +\frac{\beta R_{i}\left(x_{i}+1\right)^{-\alpha} \log ^{2}\left(x_{i}+1\right)\left(1-\left(x_{i}+1\right)^{-\alpha}\right)^{\beta-1} \log \left(1-\left(x_{i}+1\right)^{-\alpha}\right)}{1-\left(1-\left(x_{i}+1\right)^{-\alpha}\right)^{\beta}},
\end{aligned}
$$

and

$$
\begin{aligned}
\hat{l}_{\alpha \beta \beta}=\hat{l}_{\beta \alpha \beta} & =\hat{l}_{\beta \beta \alpha}=\left.\frac{\partial^{3} l}{\partial \beta^{2} \alpha}\right|_{\alpha=\hat{\alpha}, \beta=\hat{\beta}} \\
& =\sum_{i=r+1}^{m}\left(-\frac{2 \beta R_{i}\left(x_{i}+1\right)^{-\alpha} \log \left(x_{i}+1\right)\left(1-\left(x_{i}+1\right)^{-\alpha}\right)^{\beta \beta-1} \log ^{2}\left(1-\left(x_{i}+1\right)^{-\alpha}\right)}{\left(1-\left(1-\left(x_{i}+1\right)^{-\alpha}\right)^{\beta}\right)^{\beta}}\right. \\
& -\frac{2 R_{i}\left(x_{i}+1\right)^{-\alpha} \log \left(x_{i}+1\right)\left(1-\left(x_{i}+1\right)^{-\alpha}\right)^{2 \beta-1} \log \left(1-\left(x_{i}+1\right)^{-\alpha}\right)}{\left(1-\left(1-\left(x_{i}+1\right)^{-\alpha}\right)^{\beta}\right)^{2}} \\
& -\frac{3 \beta R_{i}\left(x_{i}+1\right)^{-\alpha} \log \left(x_{i}+1\right)\left(1-\left(x_{i}+1\right)^{-\alpha}\right)^{2 \beta-1} \log ^{2}\left(1-\left(x_{i}+1\right)^{-\alpha}\right)}{\left(1-\left(1-\left(x_{i}+1\right)^{-\alpha}\right)^{\beta}\right)^{2}} \\
& -\frac{2 R_{i}\left(x_{i}+1\right)^{-\alpha} \log \left(x_{i}+1\right)\left(1-\left(x_{i}+1\right)^{-\alpha}\right)^{\beta-1} \log ^{-\alpha}\left(1-\left(x_{i}+1\right)^{-\alpha}\right)}{1-\left(1-\left(x_{i}+1\right)^{-\alpha}\right)^{\beta}} \\
& -\frac{\beta R_{i}\left(x_{i}+1\right)^{-\alpha} \log \left(x_{i}+1\right)\left(1-\left(x_{i}+1\right)^{-\alpha}\right)^{\beta-1} \log ^{2}\left(1-\left(x_{i}+1\right)^{-\alpha}\right)}{1-\left(1-\left(x_{i}+1\right)^{-\alpha}\right)^{\beta}} .
\end{aligned}
$$

Using (16)

$\hat{\rho}_{\alpha}=\frac{\partial \rho}{\partial \alpha}=\frac{a_{1}-1}{\hat{\alpha}}-a_{1} b_{1} \alpha^{a_{1}-1} \quad$ and $\quad \hat{\rho}_{\beta}=\frac{\partial \rho}{\partial \beta}=\frac{a_{2}-1}{\hat{\beta}}-a_{2} b_{2} \beta^{a_{2}-1}$

Also $\sigma_{i j}$ for $i, j=1,2$. is defined by,

$\hat{\sigma}_{\alpha \alpha}=\left.\frac{-\frac{\partial^{2} l}{\partial \beta^{2}}}{\hat{\sigma}}\right|_{\alpha=\hat{\alpha}, \beta=\hat{\beta}}, \hat{\sigma}_{\beta \beta}=\left.\frac{-\frac{\partial^{2} l}{\partial \alpha^{2}}}{\hat{\sigma}}\right|_{\alpha=\hat{\alpha}, \beta=\hat{\beta}}$ and $\hat{\sigma}_{\alpha \beta}=\hat{\sigma}_{\beta \alpha}=\left.\frac{\frac{\partial^{2} l}{\partial \alpha \beta}}{\hat{\sigma}}\right|_{\alpha=\hat{\alpha}, \beta=\hat{\beta}}$,

where $\sigma$ is defined by, 


$$
\hat{\sigma}=\frac{\partial^{2} l}{\partial \alpha^{2}} \frac{\partial^{2} l}{\partial \beta^{2}}-\left.\left(\frac{\partial^{2} l}{\partial \alpha \beta}\right)^{2}\right|_{\alpha=\hat{\alpha}, \beta=\hat{\beta}} .
$$

Now, using the Lindely rule (32)to get approximate for the Bayesian estimates for the parameters $\alpha$ and $\beta$ under the squared error loss function $L_{1}$. For $\alpha$, we use $u(\alpha, \beta)=\alpha$ and $\hat{\alpha}_{B S}$ is given by,

$$
\begin{aligned}
& \hat{\alpha}_{B S}=E(\alpha \mid \underline{x}) \\
& =\hat{\alpha}+\hat{\rho}_{\alpha} \hat{\sigma}_{\alpha \alpha}+\hat{\rho}_{\beta} \hat{\sigma}_{\alpha \beta}+0.5\left[\hat{\sigma}_{\alpha \alpha}^{2} \hat{l}_{\alpha \alpha \alpha}+2 \hat{\sigma}_{\alpha \alpha} \hat{\sigma}_{\beta \alpha} \hat{l}_{\alpha \alpha \beta}+\hat{\sigma}_{\alpha \beta}^{2} \hat{l}_{\alpha \beta \beta}\right\rfloor
\end{aligned}
$$

For $\beta$, we put $u(\alpha, \beta)=\beta$ and the $\hat{\beta}_{B S}$ is given by:

$$
\begin{aligned}
& \hat{\beta}_{B S}=E(\beta \mid \underline{x}) \\
& =\hat{\beta}+\hat{\rho}_{\alpha} \hat{\sigma}_{\beta \alpha}+\hat{\rho}_{\beta} \hat{\sigma}_{\beta \beta}+0.5\left\lfloor\hat{\sigma}_{\beta \beta}^{2} \hat{l}_{\beta \beta \beta}+2 \hat{\sigma}_{\alpha \beta} \hat{\sigma}_{\beta \beta} \hat{l}_{\beta \beta \alpha}+\hat{\sigma}_{\alpha \beta}^{2} \hat{l}_{\beta \alpha \alpha}\right\rfloor
\end{aligned}
$$

Also, the Bayes estimates for $\alpha$ and $\beta$ under the LINEX loss function $L_{2}$ can be obtained as follows: for $\alpha$, we take $u(\alpha, \beta)=e^{-h \alpha}$ and $\hat{\alpha}_{B L}$ is given by,

$$
\hat{\alpha}_{B L}=-\frac{1}{h} \log E\left(e^{-h \alpha} \mid \underline{x}\right),
$$

where

$$
\begin{aligned}
& E\left(e^{-h \alpha} \mid \underline{x}\right)=e^{-h \hat{\alpha}}+0.5 h e^{-h \hat{\alpha}}\left[h \hat{\sigma}_{\alpha \alpha}-2 \hat{\rho}_{\alpha} \hat{\sigma}_{\alpha \alpha}-2 \hat{\rho}_{\beta} \hat{\sigma}_{\alpha \beta}\right] \\
& -0.5 h e^{-h \hat{\alpha}}\left[\hat{\sigma}_{\alpha \alpha}^{2} \hat{l}_{\alpha \alpha \alpha}+2 \hat{\sigma}_{\alpha \alpha} \hat{\sigma}_{\beta \alpha} \hat{l}_{\alpha \alpha \beta}+\hat{\sigma}_{\alpha \beta}^{2} \hat{l}_{\alpha \beta \beta}\right\rfloor
\end{aligned}
$$

Also, the Bayes estimate for $\beta$ under the LINEX loss function is given by,

$$
\hat{\beta}_{B L}=-\frac{1}{h} \log E\left(e^{-h \beta} \mid \underline{x}\right),
$$

where

$$
\begin{aligned}
& E\left(e^{-h \beta} \mid \underline{x}\right)=e^{-h \hat{\beta}}+0.5 h e^{-h \hat{\beta}}\left[h \hat{\sigma}_{\beta \beta}-2 \hat{\rho}_{\beta} \hat{\sigma}_{\beta \beta}-2 \hat{\rho}_{\alpha} \hat{\sigma}_{\beta \alpha}\right] \\
& -0.5 h e^{-h \hat{\beta}}\left[\hat{\sigma}_{\beta \beta}^{2} \hat{l}_{\beta \beta \beta}+2 \hat{\sigma}_{\alpha \beta} \hat{\sigma}_{\beta \beta} \hat{l}_{\beta \beta \alpha}+\hat{\sigma}_{\alpha \beta}^{2} \hat{l}_{\beta \alpha \alpha}\right]
\end{aligned}
$$

For the Bayes estimate of $\alpha$ under the generalized entropy loss function $L_{3}$, we use $u(\alpha, \beta)=\alpha^{-q}$ and $\hat{\alpha}_{B E}$ is given by,

$$
\hat{\alpha}_{B E}=E\left(\alpha^{-q} \mid \underline{x}\right)^{-1 / q} \text {, }
$$

where

$$
\begin{aligned}
& E\left(\alpha^{-q} \mid \underline{x}\right)=\hat{\alpha}^{-q}+0.5 q \alpha^{-(q+2)}\left[(q+1) \hat{\sigma}_{\alpha \alpha}-2 \hat{\alpha} \hat{\rho}_{\alpha} \hat{\sigma}_{\alpha \alpha}-2 \hat{\alpha} \hat{\rho}_{\beta} \hat{\sigma}_{\alpha \beta}\right] \\
& -0.5 q \alpha^{-(q+1)}\left[\hat{\sigma}_{\alpha \alpha}^{2} \hat{l}_{\alpha \alpha \alpha}+2 \hat{\sigma}_{\alpha \alpha} \hat{\sigma}_{\beta \alpha} \hat{l}_{\alpha \alpha \beta}+\hat{\sigma}_{\alpha \beta}^{2} \hat{l}_{\alpha \beta \beta}\right\rfloor
\end{aligned}
$$

Also, we take $u(\alpha, \beta)=\beta^{-q}$ to obtain the Bayes estimate for $\beta$ under $L_{3}$,

$$
\hat{\beta}_{B E}=E\left(\beta^{-q} \mid \underline{x}\right)^{-1 / q},
$$

where

$$
\begin{aligned}
& E\left(\beta^{-q} \mid \underline{x}\right)=\hat{\beta}^{-q}+0.5 q \beta^{-(q+2)}\left\lfloor(q+1) \hat{\sigma}_{\beta \beta}-2 \hat{\beta} \hat{\rho}_{\beta} \hat{\sigma}_{\beta \beta}-2 \hat{\beta} \hat{\rho}_{\alpha} \hat{\sigma}_{\beta \alpha}\right\rfloor \\
& -0.5 q \beta^{-(q+1)}\left[\hat{\sigma}_{\beta \beta}^{2} \hat{l}_{\beta \beta \beta}+2 \hat{\sigma}_{\alpha \beta} \hat{\sigma}_{\beta \beta} \hat{l}_{\beta \beta \alpha}+\hat{\sigma}_{\alpha \beta}^{2} \hat{l}_{\beta \alpha \alpha}\right\rfloor
\end{aligned}
$$

Finally the Bayes estimate of $\alpha$ using Al-Bayatti loss function $L_{4}$ is obtained as follows, 


$$
\hat{\alpha}_{B B}=\frac{E\left(\alpha^{c+1} \mid \underline{x}\right)}{E\left(\alpha^{c} \mid \underline{x}\right)}
$$

where

$$
\begin{gathered}
E\left(\alpha^{p} \mid \underline{x}\right)=\hat{\alpha}^{p}+0.5 p \hat{\alpha}^{p-2}\left[(p-1) \hat{\sigma}_{\alpha \alpha}+2 \hat{\alpha} \hat{\rho}_{\alpha} \hat{\sigma}_{\alpha \alpha}+2 \hat{\alpha} \hat{\rho}_{\beta} \hat{\sigma}_{\alpha \beta}\right] \\
+0.5 p \hat{\alpha}^{p-1}\left[\hat{\sigma}_{\alpha \alpha}^{2} \hat{l}_{\alpha \alpha \alpha}+2 \hat{\sigma}_{\alpha \alpha} \hat{\sigma}_{\beta \alpha} \hat{l}_{\alpha \alpha \beta}+\hat{\sigma}_{\alpha \beta}^{2} \hat{l}_{\alpha \beta \beta}\right\rfloor
\end{gathered}
$$

where $p=c, c+1$. and the Bayes estimate of $\beta$ using Al-Bayatti loss function $L_{4}$ is obtained as follows,

$$
\hat{\beta}_{B B}=\frac{E\left(\beta^{c+1} \mid \underline{x}\right)}{E\left(\beta^{c} \mid \underline{x}\right)},
$$

where

$$
\begin{aligned}
& E\left(\beta^{p} \mid \underline{x}\right)=\hat{\beta}^{p}+0.5 p \hat{\beta}^{p-2}\left\lfloor(p-1) \hat{\sigma}_{\beta \beta}+2 \hat{\beta} \hat{\rho}_{\beta} \hat{\sigma}_{\beta \beta}+2 \hat{\beta} \hat{\rho}_{\alpha} \hat{\sigma}_{\beta \alpha}\right\rfloor \\
& +0.5 p \hat{\beta}^{p-1}\left[\hat{\sigma}_{\beta \beta}^{2} \hat{l}_{\beta \beta \beta}+2 \hat{\sigma}_{\alpha \beta} \hat{\sigma}_{\beta \beta} \hat{l}_{\beta \beta \alpha}+\hat{\sigma}_{\alpha \beta}^{2} \hat{l}_{\beta \alpha \alpha}\right\rfloor
\end{aligned}
$$

and $p=c, c+1$.

\section{Two-Sample Bayesian Prediction}

In this section, we consider the Bayesian prediction of a future order statistics based on general progressively censored data $x$. Let $Y_{1} \leq Y_{2} \leq \ldots \leq Y_{w}$ are the order statistics of a future random sample of size $w$ from the same population, then the marginal density function of the $l_{\text {th }}$ order statistics $y_{l}, l=1,2, \ldots, w$, as obtained by Xiuyun and Zaizai (2016) and is given by,

$$
\begin{aligned}
f^{*}\left(y_{l} \mid \alpha, \beta\right)= & \frac{w !}{(w-1) !(l-1) !}\left[F\left(y_{l} \mid \alpha, \beta\right)\right]^{l-1}\left[1-F\left(y_{l} \mid \alpha, \beta\right)\right]^{w-l} f\left(y_{l} \mid \alpha, \beta\right) \\
& =\frac{w !}{(w-1) !(l-1) !} \sum_{l_{1}=0}^{w-l}\left(\begin{array}{c}
w-l \\
l_{1}
\end{array}\right)(-1)^{l_{1}}\left[F\left(y_{l} \mid \alpha, \beta\right)\right]^{l-1+l_{1}} f\left(y_{l} \mid \alpha, \beta\right) .
\end{aligned}
$$

Then, the Bayesian predictive density function of $y_{l}$ given $x$ is obtained as follows,

$$
f^{*}\left(y_{l} \mid \underline{x}\right)=\int_{0}^{\infty} \int_{0}^{\infty} f^{*}\left(y_{l} \mid \alpha, \beta\right) \pi(\alpha, \beta \mid \underline{x}) d \alpha d \beta .
$$

Then the predictive reliability function is established by

$$
\begin{aligned}
& R^{*}\left(y_{l} \mid \underline{x}\right)=\int_{y_{l}}^{\infty} f^{*}(z \mid \underline{x}) d z \\
& =\int_{y_{l}}^{\infty}\left(\int_{0}^{\infty} \int_{0}^{\infty} f^{*}(z \mid \alpha, \beta) \pi(\alpha, \beta \mid \underline{x}) d \alpha d \beta\right) d z \\
& =\frac{w !}{(w-l) !(l-1) !} \sum_{l_{1}=0}^{w-l}\left(\begin{array}{c}
w-l \\
l_{1}
\end{array}\right) \frac{(-1)^{l_{1}}}{l+l_{1}} H_{l_{1}}\left(y_{l} \mid \underline{x}\right),
\end{aligned}
$$

where

$$
H_{l_{1}}\left(y_{l} \mid \underline{x}\right)=\int_{0}^{\infty} \int_{0}^{\infty}\left[1-\left(F\left(y_{l} \mid \alpha, \beta\right)\right)^{l+l_{1}}\right] \pi(\alpha, \beta \mid \underline{x}) d \alpha d \beta .
$$

The integration in (55) cannot be computed analytically, so we will use Lindely method in (32) to obtain an approximate for this integration by putting $U(\alpha, \beta)$ as follows, 


$$
U(\alpha, \beta)=1-\left(F\left(y_{l} \mid \alpha, \beta\right)\right)^{l+l_{1}}=1-\left(1-\left(1+y_{l}\right)^{-\alpha}\right)^{\beta\left(l+l_{1}\right)} .
$$

Then, the $100(1-\gamma)$ Bayesian prediction bounds for $Y_{l}$ are obtained by solving the following two equations:

$$
\frac{w !}{(w-l) !(l-1) !} \sum_{l_{1}=0}^{w-l}\left(\begin{array}{c}
w-l \\
l_{1}
\end{array}\right) \frac{(-1)^{l_{1}}}{l+l_{1}} H_{l_{1}}\left(y_{l} \mid \underline{x}\right)=\left\{\begin{array}{l}
\gamma / 2, \\
1-\gamma / 2 .
\end{array}\right.
$$

\section{Illustrative Example}

Assume that we have the set of data with size $n=50$ generated from the inverted Kumaraswamy distribution with parameters $\alpha=1, \beta=2$. We assume that the number of failures $m=30$ and the number of unobserved failures $r=5$. The sample is obtained in the following table

$$
\begin{array}{llllllllll}
0.169, & 0.232, & 0.410, & 0.412, & 0.413, & 0.494, & 0.637, & 0.667, & 0.708, & 0.881, \\
1.009, & 1.174, & 1.232, & 1.299, & 1.385, & 1.413, & 1.415, & 1.540, & 1.596, & 1.627, \\
1.768, & 2.143, & 2.247, & 2.260, & 2.298, & 2.794, & 2.806, & 3.006, & 3.023, & 3.072, \\
3.194, & 3.822, & 4.146, & 4.513, & 4.820, & 4.921, & 5.284, & 5.510, & 6.027, & 6.507, \\
7.713, & 10.681, & 11.182, & 13.056, & 13.181, & 17.468, & 24.368, & 31.379, & 77.184, & 96.741 .
\end{array}
$$

\begin{tabular}{|c|c|c|c|}
\hline $\mathrm{y}$ & Scheme 1 & Scheme 2 & Scheme 3 \\
\hline & $\mathrm{L} \quad \mathrm{U}$ & L U & $\mathrm{U}$ \\
\hline$Y_{1}$ & $\begin{array}{ll}0.017 & 1.791\end{array}$ & $0.098 \quad 1.671$ & $0.017 \quad 1.890$ \\
\hline$Y_{2}$ & $\begin{array}{ll}0.099 & 2.838 \\
\end{array}$ & $0.204 \quad 2.652$ & $0.097 \quad 3.027$ \\
\hline$Y_{3}$ & $0.223 \quad 4.481$ & 0.2213 .806 & 0.2074 .696 \\
\hline$Y_{4}$ & $\begin{array}{ll}0.528 & 6.157 \\
\end{array}$ & $0.532 \quad 5.328$ & 0.3396 .995 \\
\hline$Y_{5}$ & $0.644 \quad 9.318$ & $0.621 \quad 7.455$ & $\begin{array}{ll}0.499 & 10.583\end{array}$ \\
\hline$Y_{6}$ & $0.776 \quad 14.734$ & $0.578 \quad 11.086$ & $0.705 \quad 16.925$ \\
\hline$Y_{7}$ & 1.06927 .633 & $0.843 \quad 16.929$ & $0.999 \quad 30.25$ \\
\hline$Y_{8}$ & $\begin{array}{ll}.471 & 52.910\end{array}$ & $1.232 \quad 29.619$ & $1.606 \quad 67.65$ \\
\hline$Y_{9}$ & $3.253 \quad 70.737$ & 1.79275 .688 & $1.659 \quad 259.64$ \\
\hline$Y_{10}$ & $5.095 \quad 576.807$ & $3.015 \quad 594.42$ & $1.728 \quad 7885.53$ \\
\hline
\end{tabular}

Now, this sample will used to predict with a future order statistics say $Y_{1}<Y_{2}<\ldots<Y_{w}$, where $w=10$, the prediction pounds are obtained with $\gamma=0.05$. the values of the hyper parameters are $\left(a_{1}, b_{1}\right)=(5.13,0.65)$ for $\alpha$ and $\left(a_{2}, b_{2}\right)=(10.81,0.00034)$ for $\beta$. the obtained intervals are obtained in Table 1.

Table 1: Two-sample Bayesian intervals for $y_{1}<y_{2}<\ldots<y_{10}$

\section{Simulation Study}

In this section, the performance of the proposed methods is evaluated using Monte Carlo 
simulation study. All calculations are constructed using Wolfram Mathematica 9. We will compare the MLEs and the Bayesian estimators under four loss functions, also the asymptotic confidence intervals will constructed with confidence degree $95 \%$. The samples are generated from the Inverted Kumaraswamy distribution with the parameters $\alpha$ and $\beta$, which have the following chosen real values $(\alpha, \beta)=(0.5,1)$ with sample size $n=20,50,100$ and the number of observations $m=15,20,30,50$ and 70 while the number of unobserved failures $r=3,5,10$ . Under the general progressive censoring, different schemes will used in this simulation, which given by:

1. Scheme I: $R_{i}= \begin{cases}\left\lceil\frac{2(n-m)}{m-r}\right\rceil=B, & \text { i is odd \& } n-m-\sum_{j=r+1}^{i-1} R_{j}>B ; \\ o, & \text { otherwise. }\end{cases}$ for $i=r+1, r+2, \ldots, \operatorname{Min}\left[m-1, r+\left\lfloor\frac{2(n-m)}{B}\right\rfloor\right]$ and $R_{m}=n-m-\sum_{i=r+1}^{m-1} R_{i}$.

2. Scheme II: $R_{i}= \begin{cases}\left\lceil\frac{2(n-m)}{m-r}\right\rceil=B, & i \text { is even } \& n-m-\sum_{j=r+1}^{i-1} R_{j}>B \\ o, & \text { otherwise } .\end{cases}$ for $i=r+1, r+2, \ldots, \operatorname{Min}\left[m-1, r+\left\lfloor\frac{2(n-m)}{B}\right\rfloor\right]$ and $R_{m}=n-m-\sum_{i=r+1}^{m-1} R_{i}$.

3. Scheme III: $R_{m}=n-m$ and $R_{i}=0$ for $i \neq m$

The Bayesian estimates are obtained using the Lindely method, the hyper parameters of theWeibull prior distributions for $\alpha$ and $\beta$ are taken as follows:

\begin{tabular}{|c|c|c|}
\hline Priors & Hyper parameters of $\alpha=0.5$ & Hyper parameters of $\beta=1$ \\
\hline Informative(Weibull) & $\left(a_{1}, b_{1}\right)=(2.379,3.905)$ & $\left(a_{2}, b_{2}\right)=(5.13,0.65)$ \\
\hline Informative(Exponential) & $\left(a_{1}, b_{1}\right)=(1,2)$ & $\left(a_{2}, b_{2}\right)=(1,1)$ \\
\hline Non-Informative & $\left(a_{1}, b_{1}\right)=(0,0)$ & $\left(a_{2}, b_{2}\right)=(0,0)$ \\
\hline
\end{tabular}

The Bayesian estimates are obtained using the squared error loss function SEL, the LINEX loss function with $h=1,-0.5$, the generalized entropy GE with $q=1$ and Al-Bayatti loss function with $c=0.5,-0.5$. The process of simulation will be executed 1000 times, then the average value are calculated to be the estimate value. Also we obtain the mean of 1000 lower and upper confidence limits for the asymptotic confidence intervals of the parameters with $95 \%$ confidence limits.

In Tables 2 and 3, we present the the average of estimates and mean square error (MSE) of the MLEs and the BSEs for the informative Weibull priors, while the estimates using the informative priors of the exponential special case are obtained in Tables 4 and 5. The asymptotic confidence intervals with $95 \%$ confidence degree for $\alpha=0.5$ and $\beta=1$ are included in Table 6 .

\section{Concluding remarks}

In this work, we study the estimates for the parameters of inverted Kumaraswamy distribution 
under the general progressive censored samples. The estimates are obtained using the maximum likelihood method and Bayesian method under four different types of loss functions. Two sample Bayesian prediction intervals are conducted for a future sample depending on the old sample units. According to these results of simulation and the introduced example, we can draw the following conclusions:

- The estimators that obtained from Bayesian method are very close to the real values of parameters than the estimators of Maximum likelihood method.

- The estimators that depend on samples with large size $n$ and large values of $m$ are better than those with small values.

- In most cases, the smallest MSEs are obtained under Al-Bayatti loss function with $c=-0.5$ while the MSE using the LINEX loss function with $h=1$ is less than that obtained by $h=-0.5$.

- In most cases, we noted that the Bayesian estimates using the Weibull priors are better than that obtained by exponential priors and non-informative priors.

- Small differences are noted, when we use the set of hyper parameters for informative priors and those for non-informative priors.

- the asymptotic confidence bounds contains the the MLE estimates for $\alpha$ and $\beta$, the width of the intervals become small for large values of $n$ and $m$

\section{Acknowledgments}

The authors are sincerely grateful to the referees and to the Editor-in-Chief for their many constructive comments and careful reading. 
Table 2: The MLE and Bayesian estimates for $\alpha$ and $\beta$ when $\left(a_{1}, b_{1}\right)=(2.379,3.905)$ and $\left(a_{2}, b_{2}\right)=(5.13,0.65) \quad$ (Weibull priors)

\begin{tabular}{|c|c|c|c|c|c|c|c|c|c|c|c|c|c|}
\hline \multirow{3}{*}{$n(m, r)$} & \multirow{3}{*}{ C.S } & \multicolumn{6}{|c|}{ Means and MSE for $\hat{\alpha}$} & \multicolumn{6}{|c|}{ Means and MSE for $\hat{\beta}$} \\
\hline & & \multirow{2}{*}{ BS } & \multirow{2}{*}{$\mathrm{BE}$} & \multicolumn{2}{|c|}{$\mathrm{BL}$} & \multicolumn{2}{|c|}{$\mathrm{BB}$} & \multirow{2}{*}{ BS } & \multirow{2}{*}{$\mathrm{BE}$} & \multicolumn{2}{|c|}{$\mathrm{BL}$} & \multicolumn{2}{|c|}{$\mathrm{BB}$} \\
\hline & & & & $\mathrm{h}=1$ & $\mathrm{~h}=-2$ & $\mathrm{c}=0.5$ & $c=-0.5$ & & & $\mathrm{~h}=1$ & $\mathrm{~h}=-2$ & $c=0.5$ & $c=-0.5$ \\
\hline \multirow{6}{*}{$20(15,3)$} & \multirow{2}{*}{ I } & 0.565 & 0.555 & 0.558 & 0.556 & 0.549 & 0.540 & 1.181 & 1.289 & 1.167 & 1.163 & 1.152 & 1.007 \\
\hline & & 0.0164 & 0.0085 & 0.0757 & 0.0786 & 0.2548 & 0.0076 & 0.0630 & 0.1374 & 0.0578 & 0.0711 & 0.0575 & 0.0181 \\
\hline & \multirow{2}{*}{ II } & 0.576 & 0.563 & 0.576 & 0.574 & 0.576 & 0.548 & 1.185 & 1.298 & 1.172 & 1.168 & 1.157 & 1.008 \\
\hline & & 0.0159 & 0.0095 & 0.0203 & 0.0235 & 0.0368 & 0.0082 & 0.0602 & 0.1470 & 0.0539 & 0.0650 & 0.0511 & 0.0171 \\
\hline & \multirow{2}{*}{ III } & 0.591 & 0.597 & 0.591 & 0.589 & 0.589 & 0.566 & 1.239 & 1.445 & 1.218 & 1.213 & 1.196 & 1.041 \\
\hline & & 0.0180 & 0.1482 & 0.0195 & 0.0215 & 0.0256 & 0.0110 & 0.0844 & 0.2969 & 0.0726 & 0.0907 & 0.0652 & 0.0203 \\
\hline \multirow{6}{*}{$50(20,5)$} & \multirow[b]{2}{*}{1} & 0.563 & 0.541 & 0.565 & 0.563 & 0.568 & 0.526 & 1.079 & 1.074 & 1.079 & 1.075 & 1.079 & 0.960 \\
\hline & & 0.0114 & 0.0070 & 0.0135 & 0.0165 & 0.0211 & 0.0049 & 0.0196 & 0.0185 & 0.0199 & 0.0306 & 0.0211 & 0.0100 \\
\hline & \multirow{2}{*}{ II } & 0.565 & 0.565 & 0.566 & 0.564 & 0.567 & 0.529 & 1.093 & 1.099 & 1.091 & 1.087 & 1.089 & 0.972 \\
\hline & & 0.0114 & 0.0193 & 0.0125 & 0.0148 & 0.0169 & 0.0053 & 0.0223 & 0.0232 & 0.0223 & 0.0341 & 0.0232 & 0.0091 \\
\hline & \multirow{2}{*}{ III } & 0.611 & 1.102 & 0.604 & 0.602 & 0.579 & 0.585 & 1.151 & 1.217 & 1.141 & 1.137 & 1.131 & 1.013 \\
\hline & & 0.0247 & 10.153 & 0.0363 & 0.0385 & 0.1673 & 0.0157 & 0.0410 & 0.0733 & 0.0388 & 0.0520 & 0.0393 & 0.0112 \\
\hline \multirow{6}{*}{$50(30,5)$} & \multirow{2}{*}{ I } & 0.521 & 0.489 & 0.524 & 0.522 & 0.534 & 0.497 & 1.035 & 1.009 & 1.038 & 1.035 & 1.044 & 0.946 \\
\hline & & 0.0051 & 0.0037 & 0.0056 & 0.0083 & 0.0067 & 0.0041 & 0.0079 & 0.0048 & 0.0087 & 0.0177 & 0.0098 & 0.0068 \\
\hline & \multirow{2}{*}{ II } & 0.519 & 0.488 & 0.523 & 0.521 & 0.532 & 0.496 & 1.032 & 1.007 & 1.036 & 1.033 & 1.041 & 0.944 \\
\hline & & 0.0053 & 0.0038 & 0.0057 & 0.0083 & 0.0069 & 0.0043 & 0.0091 & 0.0058 & 0.0099 & 0.0198 & 0.0110 & 0.0080 \\
\hline & \multirow{2}{*}{ III } & 0.533 & 0.499 & 0.536 & 0.534 & 0.545 & 0.506 & 1.062 & 1.043 & 1.065 & 1.061 & 1.068 & 0.954 \\
\hline & & 0.0052 & 0.0026 & 0.0058 & 0.0084 & 0.0075 & 0.0024 & 0.0118 & 0.0076 & 0.0126 & 0.0224 & 0.0139 & 0.0064 \\
\hline \multirow{6}{*}{$100(50,10)$} & \multirow{2}{*}{ I } & 0.501 & 0.477 & 0.503 & 0.501 & 0.511 & 0.484 & 0.997 & 0.975 & 1.002 & 0.998 & 1.007 & 0.942 \\
\hline & & 0.0038 & 0.0038 & 0.0039 & 0.0069 & 0.0043 & 0.0038 & 0.0052 & 0.0048 & 0.0055 & 0.0139 & 0.0058 & 0.0071 \\
\hline & II & 0.499 & 0.476 & 0.502 & 0.0501 & 0.509 & 0.483 & 0.996 & 0.974 & 1.001 & 0.997 & 1.006 & 0.942 \\
\hline & II & 0.0037 & 0.0038 & 0.0038 & 0.0060 & 0.0042 & 0.0038 & 0.0052 & 0.0055 & 0.0151 & 0.0049 & 0.0058 & 0.0071 \\
\hline & Шा & 0.508 & 0.479 & 0.511 & 0.510 & 0.521 & 0.487 & 1.015 & 0.991 & 1.018 & 1.016 & 1.024 & 0.943 \\
\hline & III & 0.0037 & 0.0032 & 0.0039 & 0.0059 & 0.0047 & 0.0034 & 0.0056 & 0.0041 & 0.0061 & 0.0147 & 0.0068 & 0.0066 \\
\hline & I & 0.495 & 0.480 & 0.497 & 0.495 & 0.502 & 0.485 & 0.987 & 0.967 & 0.991 & 0.988 & 0.995 & 0.942 \\
\hline & 1 & 0.0031 & 0.0033 & 0.0032 & 0.0054 & 0.0032 & 0.0032 & 0.0046 & 0.0049 & 0.0048 & 0.0132 & 0.0048 & 0.0068 \\
\hline & II & 0.492 & 0.478 & 0.495 & 0.492 & 0.500 & 0.483 & 0.991 & 0.971 & 0.995 & 0.992 & 0.999 & 0.946 \\
\hline $100(10,10)$ & $\pi$ & 0.0031 & 0.0033 & 0.0031 & 0.0051 & 0.0032 & 0.0032 & 0.0042 & 0.0044 & 0.0044 & 0.0127 & 0.0045 & 0.0062 \\
\hline & & 0.497 & 0.481 & 0.499 & 0.497 & 0.506 & 0.486 & 0.992 & 0.971 & 0.995 & 0.992 & 1.000 & 0.941 \\
\hline & III & 0.0031 & 0.0032 & 0.0032 & 0.0060 & 0.0034 & 0.0032 & 0.0047 & 0.0047 & 0.0048 & 0.0142 & 0.0050 & 0.0069 \\
\hline
\end{tabular}


Table 3: the MLE and Bayesian estimates for $\alpha$ and $\beta$ when $\left(a_{1}, b_{1}\right)=(1,2)$ and $\left(a_{2}, b_{2}\right)=(1,1) \quad($ Exponential priors case)

\begin{tabular}{|c|c|c|c|c|c|c|c|c|c|c|c|c|c|}
\hline \multirow{3}{*}{$\mathrm{n}(\mathrm{m}, \mathrm{r})$} & \multirow{3}{*}{ C.S } & \multicolumn{6}{|c|}{ Means and MSE for $\hat{\alpha}$} & \multicolumn{6}{|c|}{ Means and MSE for $\beta$} \\
\hline & & \multirow{2}{*}{ BS } & \multirow{2}{*}{$\mathrm{BE}$} & \multicolumn{2}{|c|}{ BL } & \multicolumn{2}{|c|}{ BB } & \multirow{2}{*}{ BS } & \multirow{2}{*}{$\mathrm{BE}$} & \multicolumn{2}{|c|}{ BL } & \multicolumn{2}{|c|}{$\mathrm{BB}$} \\
\hline & & & & $\mathrm{h}=1$ & $\mathrm{~h}=2$ & $\mathrm{c}=0.5$ & $c=0.5$ & & & $\mathrm{~h}=1$ & $\mathrm{~h}=2$ & $\mathrm{c}=0.5$ & $c=-0.5$ \\
\hline \multirow{6}{*}{$20(15,3)$} & & 0.435 & 0.439 & 0.426 & 0.424 & 0.426 & 0.431 & 1.123 & 1.014 & 1.198 & 1.193 & 1.205 & 0.798 \\
\hline & 1 & $(0.015)$ & $(0.023)$ & $(0.023)$ & $(0.025)$ & $(0.033)$ & (0.019) & $(0.129)$ & $(0.112)$ & $(0.213)$ & $(0.228)$ & $(0.183)$ & $(0.062)$ \\
\hline & II & 0.432 & 0.438 & 0.422 & 0.421 & 0.421 & 0.429 & 1.132 & 1.019 & 1.211 & 1.208 & 1.217 & 0.799 \\
\hline & 11 & $(0.015)$ & $(0.023)$ & $(0.022)$ & $(0.024)$ & $(0.032)$ & $(0.019)$ & $(0.132)$ & $(0.111)$ & $(0.219)$ & $(0.226)$ & $(0.190)$ & $(0.061)$ \\
\hline & $\pi$ & 0.402 & 0.421 & 0.383 & 0.383 & 0.377 & 0.407 & 1.132 & 1.009 & 1.213 & 1.211 & 1.225 & 0.771 \\
\hline & III & $(0.019)$ & $(0.026)$ & $(0.042)$ & $(0.043)$ & $(0.056)$ & $(0.022)$ & $(0.142)$ & $(0.114)$ & $(0.272)$ & $(0.278)$ & $(0.215)$ & $(0.072)$ \\
\hline \multirow{6}{*}{$50(20,5)$} & & 0.465 & 0.453 & 0.462 & 0.461 & 0.475 & 0.449 & 1.115 & 1.037 & 1.153 & 1.148 & 1.166 & 0.890 \\
\hline & 1 & $(0.013)$ & $(0.026)$ & $(0.020)$ & $(0.023)$ & $(0.014)$ & $(0.021)$ & $(0.122)$ & $(0.094)$ & (0.158) & $(0.174)$ & $(0.153)$ & $(0.041)$ \\
\hline & II & 0.460 & 0.453 & 0.453 & 0.452 & 0.465 & 0.447 & 1.109 & 1.031 & 1.148 & 1.144 & 1.162 & 0.878 \\
\hline & II & $(0.012)$ & $(0.026)$ & $(0.026)$ & $(0.028)$ & $(0.021)$ & $(0.021)$ & $(0.112)$ & $(0.087)$ & $(0.146)$ & $(0.159)$ & $(0.140)$ & $(0.040)$ \\
\hline & & 0.396 & 0.423 & 0.369 & 0.367 & 0.361 & 0.403 & 1.142 & 1.041 & 1.196 & 1.193 & 1.211 & 0.832 \\
\hline & III & $(0.017)$ & $(0.026)$ & $(0.051)$ & $(0.054)$ & $(0.071)$ & $(0.022)$ & $(0.171)$ & $(0.120)$ & $(0.243)$ & $(0.252)$ & $(0.227)$ & $(0.049)$ \\
\hline \multirow{6}{*}{$50(30,5)$} & I & 0.503 & 0.476 & 0.507 & 0.506 & 0.52 & 0.484 & 1.076 & 1.019 & 1.096 & 1.093 & 1.108 & 0.943 \\
\hline & 1 & $(0.014)$ & $(0.016)$ & $(0.015)$ & $(0.017)$ & $(0.015)$ & $(0.016)$ & $(0.076)$ & $(0.061)$ & $(0.088)$ & $(0.097)$ & $(0.089)$ & $(0.038)$ \\
\hline & II & 0.506 & 0.480 & 0.511 & 0.508 & 0.523 & 0.486 & 1.087 & 1.029 & 1.108 & 1.104 & 1.121 & 0.949 \\
\hline & II & $(0.015)$ & $(0.016)$ & $(0.015)$ & $(0.019)$ & $(0.015)$ & $(0.016)$ & $(0.079)$ & $(0.063)$ & $(0.092)$ & $(0.107)$ & $(0.094)$ & $(0.039)$ \\
\hline & & 0.482 & 0.457 & 0.487 & 0.485 & 0.501 & 0.463 & 1.085 & 1.017 & 1.109 & 1.105 & 1.124 & 0.916 \\
\hline & III & $(0.014)$ & $(0.018)$ & $(0.014)$ & $(0.017)$ & $(0.013)$ & $(0.017)$ & $(0.092)$ & $(0.072)$ & $(0.109)$ & $(0.123)$ & $(0.109)$ & $(0.042)$ \\
\hline \multirow{6}{*}{$100(50,10)$} & $\mathrm{J}$ & 0.502 & 0.481 & 0.506 & 0.504 & 0.515 & 0.487 & 1.029 & 1.007 & 1.049 & 1.046 & 1.057 & 0.965 \\
\hline & 1 & $(0.011)$ & $(0.011)$ & $(0.011)$ & $(0.013)$ & $(0.011)$ & (0.011) & $(0.029)$ & $(0.034)$ & $(0.042)$ & $(0.051)$ & $(0.043)$ & $(0.026)$ \\
\hline & II & 0.507 & 0.486 & 0.511 & 0.509 & 0.520 & 0.493 & 1.047 & 1.014 & 1.057 & 1.054 & 1.064 & 0.973 \\
\hline & 11 & $(0.011)$ & $(0.011)$ & $(0.011)$ & $(0.014)$ & $(0.012)$ & $(0.011)$ & $(0.039)$ & $(0.034)$ & $(0.043)$ & $(0.054)$ & $(0.044)$ & $(0.027)$ \\
\hline & मा & 0.496 & 0.473 & 0.501 & 0.499 & 0.512 & 0.479 & 1.058 & 1.017 & 1.071 & 1.068 & 1.080 & 0.956 \\
\hline & 1111 & $(0.012)$ & $(0.013)$ & $(0.012)$ & $(0.014)$ & $(0.012)$ & $(0.013)$ & $(0.049)$ & $(0.042)$ & $(0.054)$ & $(0.062)$ & $(0.056)$ & $(0.029)$ \\
\hline \multirow{6}{*}{$100(70,10)$} & J & 0.501 & 0.486 & 0.503 & 0.502 & 0.508 & 0.491 & 1.029 & 1.003 & 1.036 & 1.034 & 1.043 & 0.973 \\
\hline & 1 & $(0.007)$ & $(0.007)$ & $(0.007)$ & $(0.009)$ & $(0.007)$ & $(0.007)$ & $(0.027)$ & $(0.025)$ & $(0.028)$ & $(0.038)$ & $(0.029)$ & $(0.021)$ \\
\hline & III & 0.503 & 0.488 & 0.504 & 0.503 & 0.510 & 0.493 & 1.037 & 1.011 & 1.045 & 1.041 & 1.051 & 0.981 \\
\hline & II & $(0.007)$ & $(0.007)$ & $(0.007)$ & $(0.010)$ & $(0.007)$ & $(0.007)$ & $(0.029)$ & $(0.026)$ & $(0.031)$ & $(0.042)$ & $(0.032)$ & $(0.022)$ \\
\hline & & 0.496 & 0.473 & 0.501 & 0.499 & 0.512 & 0.479 & 1.058 & 1.017 & 1.071 & 1.068 & 1.080 & 0.956 \\
\hline & III & $(0.012)$ & $(0.013)$ & $(0.012)$ & $(0.014)$ & $(0.012)$ & $(0.013)$ & $(0.049)$ & $(0.042)$ & $(0.054)$ & $(0.062)$ & $(0.056)$ & $(0.029)$ \\
\hline
\end{tabular}


Table 4: the MLE and Bayesian estimates for $\alpha$ and $\beta$ when $\left(a_{1}, b_{1}\right)=(0,0)$ and $\left(a_{2}, b_{2}\right)=(0,0)$ (Non-Informative case).

\begin{tabular}{|c|c|c|c|c|c|c|c|c|c|c|c|c|c|}
\hline \multirow{3}{*}{$\mathrm{n}(\mathrm{m}, \mathrm{r})$} & \multirow{3}{*}{ C.S } & \multicolumn{6}{|c|}{ Means and MSE for $\hat{\alpha}$} & \multicolumn{6}{|c|}{ Means and MSE for $\hat{\beta}$} \\
\hline & & \multirow{2}{*}{ BS } & \multirow{2}{*}{$\mathrm{BE}$} & \multicolumn{2}{|c|}{ BL } & \multicolumn{2}{|c|}{ BB } & \multirow{2}{*}{ BS } & \multirow{2}{*}{$\mathrm{BE}$} & \multicolumn{2}{|c|}{$\mathrm{BL}$} & \multicolumn{2}{|c|}{$\mathrm{BB}$} \\
\hline & & & & $\mathrm{h}=1$ & $h=-2$ & $c=0.5$ & $c=-0.5$ & & & $\mathrm{~h}=1$ & $\mathrm{~h}=-2$ & $c=0.5$ & $c=-0.5$ \\
\hline \multirow{3}{*}{$20(15,3)$} & I & $\begin{array}{c}0.501 \\
(0.036)\end{array}$ & $\begin{array}{c}0.469 \\
(0.039)\end{array}$ & $\begin{array}{c}0.511 \\
(0.038)\end{array}$ & $\begin{array}{c}0.508 \\
(0.043)\end{array}$ & $\begin{array}{c}0.531 \\
(0.038)\end{array}$ & $\begin{array}{c}0.475 \\
(0.038)\end{array}$ & $\begin{array}{c}1.394 \\
(1.110)\end{array}$ & $\begin{array}{c}1.193 \\
(0.706)\end{array}$ & $\begin{array}{c}1.457 \\
(1.185)\end{array}$ & $\begin{array}{c}1.451 \\
(1.209)\end{array}$ & $\begin{array}{c}1.493 \\
(1.275)\end{array}$ & $\begin{array}{r}0.890 \\
(0.084)\end{array}$ \\
\hline & II & $\begin{array}{c}0.503 \\
(0.034)\end{array}$ & $\begin{array}{c}0.471 \\
(0.037)\end{array}$ & $\begin{array}{c}0.512 \\
(0.035)\end{array}$ & $\begin{array}{c}0.511 \\
(0.037)\end{array}$ & $\begin{array}{r}0.533 \\
(0.025) \\
\end{array}$ & $\begin{array}{r}0.477 \\
(0.035)\end{array}$ & $\begin{array}{c}1.389 \\
(1.092)\end{array}$ & $\begin{array}{c}1.192 \\
(0.689)\end{array}$ & $\begin{array}{c}1.453 \\
(1.159)\end{array}$ & $\begin{array}{r}1.449 \\
(1.168)\end{array}$ & $\begin{array}{r}1.489 \\
(1.253)\end{array}$ & $\begin{array}{c}0.885 \\
(0.084)\end{array}$ \\
\hline & III & $\begin{array}{c}0.479 \\
(0.032) \\
\end{array}$ & $\begin{array}{c}0.459 \\
(0.041) \\
\end{array}$ & $\begin{array}{c}0.483 \\
(0.034) \\
\end{array}$ & $\begin{array}{c}0.482 \\
(0.034) \\
\end{array}$ & $\begin{array}{c}0.502 \\
(0.031) \\
\end{array}$ & $\begin{array}{r}0.459 \\
(0.036) \\
\end{array}$ & $\begin{array}{r}1.584 \\
(3.353) \\
\end{array}$ & $\begin{array}{c}1.788 \\
(15.302)\end{array}$ & $\begin{array}{c}1.615 \\
(2.281) \\
\end{array}$ & $\begin{array}{r}1.614 \\
(2.386) \\
\end{array}$ & $\begin{array}{r}1.676 \\
(3.034) \\
\end{array}$ & $\begin{array}{r}0.876 \\
(0.095) \\
\end{array}$ \\
\hline \multirow{3}{*}{$50(20,5)$} & I & $\begin{array}{c}0.514 \\
(0.042)\end{array}$ & $\begin{array}{c}0.473 \\
(0.044)\end{array}$ & $\begin{array}{c}0.526 \\
(0.045)\end{array}$ & $\begin{array}{c}0.525 \\
(0.046)\end{array}$ & $\begin{array}{c}0.551 \\
(0.045)\end{array}$ & $\begin{array}{c}0.481 \\
(0.044)\end{array}$ & $\begin{array}{c}1.179 \\
(0.299)\end{array}$ & $\begin{array}{c}1.083 \\
(0.211)\end{array}$ & $\begin{array}{c}1.213 \\
(0.329)\end{array}$ & $\begin{array}{c}1.211 \\
(0.245)\end{array}$ & $\begin{array}{c}1.232 \\
(0.346)\end{array}$ & $\begin{array}{c}0.925 \\
(0.067)\end{array}$ \\
\hline & II & $\begin{array}{c}0.508 \\
(0.036)\end{array}$ & $\begin{array}{c}0.467 \\
(0.039) \\
\end{array}$ & $\begin{array}{r}0.520 \\
(0.039)\end{array}$ & $\begin{array}{c}0.519 \\
(0.040) \\
\end{array}$ & $\begin{array}{r}0.546 \\
(0.039) \\
\end{array}$ & $\begin{array}{r}0.475 \\
(0.039)\end{array}$ & $\begin{array}{c}1.196 \\
(0.327) \\
\end{array}$ & $\begin{array}{c}1.095 \\
(0.241)\end{array}$ & $\begin{array}{c}1.231 \\
(0.377)\end{array}$ & $\begin{array}{c}1.228 \\
(0.383)\end{array}$ & $\begin{array}{l}1.250 \\
(0.388)\end{array}$ & $\begin{array}{r}0.921 \\
(0.069)\end{array}$ \\
\hline & III & $\begin{array}{c}0.467 \\
(0.032)\end{array}$ & $\begin{array}{c}0.438 \\
(0.044)\end{array}$ & $\begin{array}{c}0.475 \\
(0.034)\end{array}$ & $\begin{array}{c}0.474 \\
(0.037)\end{array}$ & $\begin{array}{c}0.502 \\
(0.029)\end{array}$ & $\begin{array}{c}0.437 \\
(0.040)\end{array}$ & $\begin{array}{c}1.265 \\
(0.790)\end{array}$ & $\begin{array}{c}1.589 \\
(20.75)\end{array}$ & $\begin{array}{c}1.295 \\
(0.675)\end{array}$ & $\begin{array}{r}1.293 \\
(0.681) \\
\end{array}$ & $\begin{array}{c}1.323 \\
(0.759)\end{array}$ & $\begin{array}{r}0.878 \\
(0.078)\end{array}$ \\
\hline \multirow{3}{*}{$50(30,5)$} & I & $\begin{array}{c}0.516 \\
(0.019)\end{array}$ & $\begin{array}{c}0.486 \\
(0.018)\end{array}$ & $\begin{array}{c}0.522 \\
(0.020)\end{array}$ & $\begin{array}{c}0.520 \\
(0.022)\end{array}$ & $\begin{array}{c}0.535 \\
(0.021)\end{array}$ & $\begin{array}{r}0.495 \\
(0.019)\end{array}$ & $\begin{array}{c}1.112 \\
(0.119) \\
\end{array}$ & $\begin{array}{c}1.049 \\
(0.091)\end{array}$ & $\begin{array}{c}1.132 \\
(0.134)\end{array}$ & $\begin{array}{c}1.129 \\
(0.143)\end{array}$ & $\begin{array}{l}1.145 \\
(0.137)\end{array}$ & $\begin{array}{r}0.969 \\
(0.053)\end{array}$ \\
\hline & II & $\begin{array}{c}0.507 \\
(0.020)\end{array}$ & $\begin{array}{c}0.477 \\
(0.019)\end{array}$ & $\begin{array}{c}0.512 \\
(0.021)\end{array}$ & $\begin{array}{c}0.510 \\
(0.024)\end{array}$ & $\begin{array}{c}0.526 \\
(0.021)\end{array}$ & $\begin{array}{r}0.486 \\
(0.020)\end{array}$ & $\begin{array}{c}1.088 \\
(0.112)\end{array}$ & $\begin{array}{r}1.027 \\
(0.087)\end{array}$ & $\begin{array}{c}1.107 \\
(0.125)\end{array}$ & $\begin{array}{c}1.104 \\
(0.136)\end{array}$ & $\begin{array}{l}1.121 \\
(0.127)\end{array}$ & $\begin{array}{r}0.949 \\
(0.053)\end{array}$ \\
\hline & III & $\begin{array}{c}0.510 \\
(0.024)\end{array}$ & $\begin{array}{c}.478 \\
(0.025)\end{array}$ & $\begin{array}{c}0.516 \\
(0.026)\end{array}$ & $\begin{array}{c}0.515 \\
(0.028)\end{array}$ & $\begin{array}{c}0.533 \\
(0.026)\end{array}$ & $\begin{array}{c}0.488 \\
(0.025)\end{array}$ & $\begin{array}{c}1.146 \\
(0.194) \\
\end{array}$ & $\begin{array}{c}1.070 \\
(0.145)\end{array}$ & $\begin{array}{c}1.171 \\
(0.217)\end{array}$ & $\begin{array}{c}1.167 \\
(0.232)\end{array}$ & $\begin{array}{c}1.186 \\
(0.222)\end{array}$ & $\begin{array}{c}0.955 \\
(0.063)\end{array}$ \\
\hline \multirow{3}{*}{$100(50,10)$} & I & $\begin{array}{c}0.511 \\
(0.015)\end{array}$ & $\begin{array}{c}0.488 \\
(0.014)\end{array}$ & $\begin{array}{c}0.514 \\
(0.015)\end{array}$ & $\begin{array}{c}0.513 \\
(0.018)\end{array}$ & $\begin{array}{c}0.524 \\
(0.016)\end{array}$ & $\begin{array}{r}0.495 \\
(0.015)\end{array}$ & $\begin{array}{c}1.053 \\
(0.051)\end{array}$ & $\begin{array}{c}1.019 \\
(0.044)\end{array}$ & $\begin{array}{c}1.062 \\
(0.054)\end{array}$ & $\begin{array}{c}1.059 \\
(0.063)\end{array}$ & $\begin{array}{c}1.070 \\
(0.055)\end{array}$ & $\begin{array}{r}0.976 \\
(0.033)\end{array}$ \\
\hline & II & $\begin{array}{c}0.517 \\
(0.015) \\
\end{array}$ & $\begin{array}{c}0.495 \\
(0.014) \\
\end{array}$ & $\begin{array}{r}0.521 \\
(0.015) \\
\end{array}$ & $\begin{array}{c}0.520 \\
(0.017) \\
\end{array}$ & $\begin{array}{r}0.531 \\
(0.016) \\
\end{array}$ & $\begin{array}{r}0.502 \\
(0.015) \\
\end{array}$ & $\begin{array}{c}1.057 \\
(0.046) \\
\end{array}$ & $\begin{array}{r}1.024 \\
(0.039) \\
\end{array}$ & $\begin{array}{c}1.067 \\
(0.049) \\
\end{array}$ & $\begin{array}{c}1.065 \\
(0.056) \\
\end{array}$ & $\begin{array}{l}1.075 \\
(0.051) \\
\end{array}$ & $\begin{array}{r}0.982 \\
(0.030) \\
\end{array}$ \\
\hline & III & $\begin{array}{c}0.507 \\
(0.017)\end{array}$ & $\begin{array}{c}0.481 \\
(0.017)\end{array}$ & $\begin{array}{r}0.512 \\
(0.018)\end{array}$ & $\begin{array}{c}0.511 \\
(0.020)\end{array}$ & $\begin{array}{l}0.524 \\
(0.018)\end{array}$ & $\begin{array}{r}0.489 \\
(0.017)\end{array}$ & $\begin{array}{c}1.077 \\
(0.069)\end{array}$ & $\begin{array}{c}1.034 \\
(0.057)\end{array}$ & $\begin{array}{c}1.090 \\
(0.075)\end{array}$ & $\begin{array}{c}1.087 \\
(0.083)\end{array}$ & $\begin{array}{l}1.099 \\
(0.077)\end{array}$ & $\begin{array}{c}0.971 \\
(0.039)\end{array}$ \\
\hline \multirow{3}{*}{$100(70,10)$} & I & $\begin{array}{c}0.501 \\
(0.008)\end{array}$ & $\begin{array}{c}0.486 \\
(0.008)\end{array}$ & $\begin{array}{l}0.503 \\
(0.008)\end{array}$ & $\begin{array}{c}0.501 \\
(0.011)\end{array}$ & $\begin{array}{c}0.508 \\
(0.008)\end{array}$ & $\begin{array}{l}0.491 \\
(0.008)\end{array}$ & $\begin{array}{c}1.039 \\
(0.032)\end{array}$ & $\begin{array}{l}1.012 \\
(0.029)\end{array}$ & $\begin{array}{c}1.046 \\
(0.035)\end{array}$ & $\begin{array}{r}1.043 \\
(0.045)\end{array}$ & $\begin{array}{l}1.053 \\
(0.035)\end{array}$ & $\begin{array}{l}0.982 \\
(0.025)\end{array}$ \\
\hline & II & $\begin{array}{c}0.502 \\
(0.008) \\
\end{array}$ & $\begin{array}{c}0.488 \\
(0.008) \\
\end{array}$ & $\begin{array}{c}0.504 \\
(0.008) \\
\end{array}$ & $\begin{array}{c}0.503 \\
(0.012) \\
\end{array}$ & $\begin{array}{c}0.510 \\
(0.008) \\
\end{array}$ & $\begin{array}{r}0.493 \\
(0.008)\end{array}$ & $\begin{array}{c}1.036 \\
(0.036) \\
\end{array}$ & $\begin{array}{c}1.009 \\
(0.033) \\
\end{array}$ & $\begin{array}{c}.043 \\
(0.038) \\
\end{array}$ & $\begin{array}{c}1.040 \\
(0.049) \\
\end{array}$ & $\begin{array}{c}1.049 \\
(0.039) \\
\end{array}$ & $\begin{array}{c}0.979 \\
(0.027) \\
\end{array}$ \\
\hline & III & $\begin{array}{c}0.506 \\
(0.009)\end{array}$ & $\begin{array}{c}0.491 \\
(0.009)\end{array}$ & $\begin{array}{r}0.508 \\
(0.009)\end{array}$ & $\begin{array}{c}0.507 \\
(0.012)\end{array}$ & $\begin{array}{c}0.515 \\
(0.010)\end{array}$ & $\begin{array}{r}0.496 \\
(0.009)\end{array}$ & $\begin{array}{c}1.052 \\
(0.043)\end{array}$ & $\begin{array}{c}1.022 \\
(0.037)\end{array}$ & $\begin{array}{c}1.060 \\
(0.045)\end{array}$ & $\begin{array}{c}1.056 \\
(0.057)\end{array}$ & $\begin{array}{c}1.067 \\
(0.046)\end{array}$ & $\begin{array}{r}0.985 \\
(0.020)\end{array}$ \\
\hline
\end{tabular}


Table 5: MLE and the asymptotic confidence bounds for $\alpha=0.5$ and $\beta=1$

\begin{tabular}{|c|c|c|c|cc|cc|}
\hline \multirow{2}{*}{$\mathrm{n}(\mathrm{m}, \mathrm{r})$} & \multirow{2}{*}{ C.S } & MLE of $\alpha$ & MLE of $\beta$ & \multicolumn{2}{|c|}{ Asymp. C.I for $\alpha$} & Asymp. C.I for $\beta$ \\
\cline { 3 - 8 } & & $\alpha_{M L}(M S E)$ & $\beta_{M L}(M S E)$ & $L_{\alpha}$ & $U_{\alpha}$ & $L_{\beta}$ & $U_{\beta}$ \\
\hline \multirow{3}{*}{$20(15,3)$} & I & $0.624(0.081)$ & $1.291(0.454)$ & 0.178 & 1.071 & 0.268 & 2.214 \\
& II & $0.627(0.084)$ & $1.299(0.449)$ & 0.176 & 1.077 & 0.264 & 2.334 \\
& III & $0.628(0.092)$ & $1.297(0.462)$ & 0.156 & 1.099 & 0.224 & 2.370 \\
\hline \multirow{5}{*}{$50(20,5)$} & I & $0.631(0.092)$ & $1.210(0.231)$ & 0.170 & 1.092 & 0.492 & 1.928 \\
& II & $0.643(0.099)$ & $1.209(0.225)$ & 0.163 & 1.125 & 0.478 & 1.940 \\
& III & $0.659(0.115)$ & $1.247(0.294)$ & 0.115 & 1.205 & 0.411 & 2.085 \\
\hline \multirow{5}{*}{$50(30,5)$} & I & $0.560(0.031)$ & $1.111(0.105)$ & 0.263 & 0.856 & 0.581 & 1.641 \\
& II & $0.567(0.033)$ & $1.124(0.108)$ & 0.265 & 0.868 & 0.583 & 1.665 \\
& III & $0.565(0.038)$ & $1.123(0.127)$ & 0.234 & 0.895 & 0.538 & 1.707 \\
\hline \multirow{5}{*}{$100(50,10)$} & I & $0.534(0.016)$ & $1.056(0.046)$ & 0.297 & 0.770 & 0.680 & 1.432 \\
& II & $0.539(0.017)$ & $1.064(0.046)$ & 0.303 & 0.776 & 0.686 & 1.442 \\
& III & $0.546(0.023)$ & $1.077(0.058)$ & 0.273 & 0.820 & 0.647 & 1.507 \\
\hline \multirow{3}{*}{$100(70,10)$} & I & $0.519(0.008)$ & $1.038(0.030)$ & 0.339 & 0.699 & 0.708 & 1.367 \\
& II & $0.522(0.009)$ & $1.046(0.032)$ & 0.342 & 0.701 & 0.714 & 1.378 \\
& III & $0.529(0.011)$ & $1.059(0.038)$ & 0.336 & 0.722 & 0.705 & 1.414 \\
\hline
\end{tabular}

\section{References}

1. Al-Bayyati, H. (2002). Comparing methods of estimating Weibull failure models using simulation.Unpublished $\mathrm{PhD}$ thesis.

2. AL-Fattah, A., E-Helbawy, A., and AL-Dayian, G. (2017). Inverted Kumaraswamy distribution: Properties and estimation. Pakistan Journal of Statistics 33, 1.

3. Balakrishnan, N. (2007). Progressive censoring methodology: an appraisal. Test 16, 2, 211-259.

4. Balakrishnan, N., and Aggarwala, R. (2000). Progressive censoring: theory, methods and applications. Springer Science \& Business Media.

5. El-Din, M. M., Abdel-Aty, Y., and Abu-Moussa, M. (2017). Statistical inference for theGompertz distribution based on type-II progressively hybrid censored data. Communicationsin Statistics-Simulation and Computation, 46:8, 6242-6260.

6. El-Din, M. M., Abdel-Aty, Y., and Shafay, A. (2011). Bayesian prediction for orderstatistics from a general class of distributions based on left type-ii censored data.International Journal of Mathematics \& Computation 13, D11, 34-42.

7. El-Din, M. M., Sadek, A., El-Din, M. M. M., and Sharawy, A. (2017). Characterization for Gompertz distribution based on general progressively type-ii right censored orderstatistics. International Journal of Advanced Statistics and Probability 5, 1, 52-56. 
8. El-Din, M. M., and Shafay, A. (2013). One-and two-sample Bayesian prediction intervals based on progressively type-II censored data. Statistical Papers 54, 2, 287-307.

9. Lindley, D. V. 1 (1980). Approximate Bayesian methods. Trabajos de estadística y de investigaciónoperativa 31, 223-245.

10. Mohie El-Din, M., Abdel-Aty, Y., and Shafay, A. (2012). Two-sample Bayesian predictionintervals of generalized order statistics based on multiply type-II censored data.Communications in Statistics - Theory and Methods 41, 3, 381-392.

11. Shafay, A., and Balakrishnan, N. (2012), One-and two-sample Bayesian prediction intervals based on Type-I hybrid censored data. Communications in Statistics Simulationand Computation 41, 1, 65-88.

12. Soliman, A. A. (2008). Estimations for Pareto model using general progressive censored dataand asymmetric loss. Communications in Statistics-Theory and Methods 37, 9, 1353-1370.

13. Xiuyun, P., and Zaizai, Y. (2016). Bayesian estimation and prediction for the inverse Weibulldistribution under general progressive censoring. Communications in Statistics Theoryand Methods 45, 3, 621-635. 